\section{La Révolution française}

Cahiers de l'Institut d'histoire de la Révolution française

$5 \mid 2013$

Le républicanisme anglais dans la France des

Lumières et de la Révolution

\title{
La république anglaise du Thermidor au premier anniversaire de Brumaire (1794-1800)
}

Daniele Di Bartolomeo

\section{(2) OpenEdition}

Journals

Edizione digitale

URL: http://journals.openedition.org//rf/1060

DOI: $10.4000 /$ Irf. 1060

ISSN: 2105-2557

Editore

IHMC - Institut d'histoire moderne et contemporaine (UMR 8066)

Notizia bibliografica digitale

Daniele Di Bartolomeo, « La république anglaise du Thermidor au premier anniversaire de Brumaire

(1794-1800) », La Révolution française [En ligne], 5 | 2013, mis en ligne le 31 décembre 2013, consulté

le 22 avril 2019. URL : http://journals.openedition.org//rf/1060 ; DOI : 10.4000/Irf.1060

Questo documento è stato generato automaticamente il 22 aprile 2019.

(c) La Révolution française 


\title{
La république anglaise $d u$ Thermidor au premier anniversaire de Brumaire (1794-1800)
}

\author{
Daniele Di Bartolomeo
}

\section{Introduction : L'Usage des mots (et) de l'histoire}

«Je sais bien que les dupeurs dupés me diront, ne

fût-ce que pour l'intérêt de leur orgueil : en révolution il ne faut jamais regarder en arrière. Cela veut-t-il dire qu'en révolution, l'avenir est tout, le présent pas grand-chose et le passé rien? Qu'on me montre la force humaine qui, même en révolution, pourrait séparer les uns des autres le passé, le présent et l'avenir » Le procès des 31 mai, $1^{\text {er }}$ et 2 juin, ou la défense des 71 représentans du peuple par MichelEdme Petit, Paris, 1795, p. 9

1 Le parole hanno una storia non solo perché il loro significato cambia nel tempo, ma anche poiché alludono ad atmosfere del passato ${ }^{1}$. I termini repubblica o repubblicanesimo, ad esempio, sono concetti densi di immagini storiche, che rinviano ai grandi cicli rivoluzionari del mondo antico e alle loro repliche moderne, come quella verificatasi nel XVII secolo in Inghilterra.

2 In questo articolo mostreremo come l'epopea repubblicana inglese, un repertorio di eventi, protagonisti, idee ed istituzioni esemplari, sia stata usata dai francesi nel periodo che va dalla caduta di Robespierre al primo anniversario della salita al potere di Napoleone. 
Come è noto, i patrioti francesi ed i loro avversari avevano fatto ricorso alla storia delle rivoluzioni inglesi per pensare l'attualità e le sue possibili evoluzioni sin dal $1788^{2}$. Più in generale, sappiamo come le repubbliche e i pensatori repubblicani del passato abbiamo rappresentato per i francesi ${ }^{3}$, in successione, un contraltare (positivo o negativo) della monarchia ${ }^{4}$, un repertorio per emendare in senso democratico il nascente sistema costituzionale, un'alternativa concreta (seppur insidiosa) a tale forma di governo e, infine, lo specchio di un'esperienza politica finalmente repubblicana ${ }^{5}$.

4 Si tratta di un uso delle parole (e) del passato ereditato da chi nei secoli precedenti aveva ragionato attorno all'attualità teorica e politica delle repubbliche antiche e, soprattutto, da chi come gli inglesi nel Seicento, gli americani nei decenni precedenti e gli stessi rivoluzionari dall'Ottantanove in poi lo aveva fatto nel bel mezzo di una rivoluzione.

5 L'adozione di questa prospettiva, centrata più sulla storia delle repubbliche e dei repubblicani del passato che sul loro vocabolario politico, ci consentirà di integrare $\mathrm{e}$ approfondire le recenti acquisizioni della storiografia sul ruolo del repubblicanesimo (inglese) nella Rivoluzione francese. Da qualche tempo, infatti, gli storici hanno iniziato a studiarla non solo come l'origine della politica moderna, ma anche come un laboratorio dove sono transitate le esperienze del passato e sono state discusse le suggestioni dell'attualità'. Questa svolta ha aperto nuovi cantieri di ricerca, mettendo proficuamente in discussione il topos storiografico l'eccezionalità francese, ricollocando la Rivoluzione in una prospettiva spazio-temporale ${ }^{7}$ ed evidenziando la centralità delle esperienze repubblicane presenti e passate nella costruzione dell'immaginario politico francese di fine Settecento ${ }^{8}$. Essa, inoltre, ha stimolato molti interpreti ad estendere conseguentemente alla Francia rivoluzionaria gli studi sugli itinerari medievali e moderni della tradizione repubblicana classica ${ }^{9}$, inaugurati da John Pocock e variamente declinati da Quentin Skinner, Philip Petitt e da tanti altri ${ }^{10}$.

6 Il ritardo con cui gli specialisti della Rivoluzione si sono interessati al riuso del vocabolario repubblicano classico così come è stato reinventato nell'Italia dei Comuni $\mathrm{e}$ dell'umanesimo civico, nell'Inghilterra del Seicento e negli Stati Uniti è dovuto alla convinzione che l'evento francese avesse attuato una tabula rasa, scartando la possibilità di declinare l'impresa rivoluzionaria seguendo la via (anch'essa inglese) della reinvenzione di una tradizione risalente ${ }^{11}$. Da questo punto di vista, la Rivoluzione è apparsa a qualcuno come un'occasione mancata da chi non ha saputo mettere la propria storia al servizio del cambiamento, da chi non è riuscito a costruire una via nazionale nell'alveo del repubblicanesimo ${ }^{12}$. Oltre che all'assenza di una tradizione repubblicana, il fallimento della Rivoluzione è stato specularmente addebitato alla prevalenza di un certo tipo di linguaggio repubblicano (quello classico). Un'impostazione, quest'ultima, che riposa su una schematizzazione forzata del panorama fazionale della Rivoluzione in base alla distinzione canonica tra un repubblicanesimo degli antichi (impersonato dai Giacobini) e uno dei moderni (incarnato dai Girondini) ${ }^{13}$. Non meno manichea è l'idea di chi descrive la Francia del Direttorio come l'avvento di una nuova forma di « repubblicanesimo democratico » compatibile con la modernità ${ }^{14} \mathrm{e}$ di chi, all'opposto, vede nel Termidoro la liquidazione di un repubblicanesimo fondato sull'esaltazione della sovranità popolare a vantaggio di un'altra sua declinazione, certo più moderata, ma pur sempre classica, ispirata all'idea antica di costituzione mista ${ }^{15}$. Mentre Keith Baker, infatti, sostiene che il repubblicanesimo democratico dei Giacobini abbia indotto l'adozione di una politica repressiva, similmente, Andrew Jainchill ritiene che la prognosi classica abbia incentivato i Termidoriani ad assumere un rimedio altrettanto risaliente, 
che a sua volta si è rivelato, se non controproducente, almeno inefficace ${ }^{16}$. Più propriamente, si è fatto notare come tra queste alternative ne esista almeno un'altra, impersonata dai Cordiglieri, che si ispirava al repubblicanesimo primo-moderno maturato durante e a seguito della rivoluzione inglese ${ }^{17}$.

Da questi studi emerge l'immagine di una Rivoluzione fortemente condizionata dalla competizione o dalla successione cronologica tra diversi discorsi repubblicani, dall'uso, dall'abuso o dal mancato uso di storie e parole del passato. Un'interpretazione che, a dire il vero, gli storici hanno ereditato più o meno consapevolmente dai quei superstiti del Terrore che, a partire dall'anno III, hanno imputato il fallimento della Rivoluzione all'abuso delle parole (e) della storia ${ }^{18}$.

La storiografia, quindi, pur avendo finora colmato un vuoto (segnalato dallo stesso Pocock ${ }^{19}$ ), ricostruendo (almeno in parte) le versioni rivoluzionarie del repubblicanesimo ${ }^{20}$, ha lasciato in ombra il peso esercitato sull'immaginario politico della Rivoluzione, in generale, e della Repubblica direttoriale, in particolare, dalle vicissitudini della repubblica e dei repubblicani (inglesi). Il repubblicanesimo, classico o moderno che sia, è un discorso attorno alla contingenza dei regimi politici e ai mezzi ideati e messi in pratica nel passato per fondarli ed evitarne la decadenza, per giustificarli o delegittimarli ${ }^{21}$. Il discorso repubblicano è insieme una diagnosi, una prognosi e una lista di rimedi (spesso auspicati, ma a volte anche contestati) ricavati dall'analisi dei fatti del passato o dalle riflessioni scaturite a commento di essi e magari già sperimentati (materialmente o solo teoricamente) altrove nel tempo e nello spazio ${ }^{22}$. Ecco perché, ad esempio, i patrioti francesi sono ricorsi alla versione storica di Catharine Macaulay come un "antidoto repubblicano", per smentire la nefasta profezia che i controrivoluzionari avevano ricavato dalla lettura della Storia dell'Inghilterra di David Hume ${ }^{23}$. La storia delle repubbliche, quindi, non è solo un'immagine stilizzata al servizio di un discorso politico atterrito dal rischio della decadenza e ossessionato dagli imperativi della virtù e della trasparenza ${ }^{24}$, ma è anche un repertorio concreto di azioni ed esiti di cui gli attori storici si servono in modo disinvolto ${ }^{25}$.

\section{L'an III : le temps des bilans}

$$
\begin{array}{r}
\text { « Robespierre aussi avait poursuivi des traitres, et } \\
\text { Robespierre fut un lâche tyran. Cromwel avait } \\
\text { chassé les Stuarts, et son joug prépara les Anglais } \\
\text { au retour de leurs rois " } \\
\text { Les citoyens d'Arras, réunis en assemblée générale, } \\
\text { à la Convention nationale, 24 thermidor an II [11 agosto }
\end{array}
$$

Alla vigilia della caduta di Robespierre, il 26 luglio 1794 (8 termidoro anno II) ${ }^{26}$, nel consueto supplemento dedicato alla lettura, il Moniteur segnala che in una libreria della capitale sono in vendita, tra le altre cose, le traduzioni di tre vecchi libri di storia: l' Histoire de la république romaine di Sallustio, l'Histoire de l'Angleterre di Hume e l'Histoire de la décadence et de la chute de l'Empire Romain di Gibbon ${ }^{27}$.

Chi quel giorno avesse deciso di recarsi presso il libraio Servière a spendere duecentoventi lire, l'indomani si sarebbe trovato tra le mani quelli che noi chiameremmo degli instant books profetici. Testi capaci di spiegare i fatti straordinari appena accaduti 
come l'ultima incarnazione della grande epopea classica, di cui gli inglesi avevano offerto la replica più ravvicinata.

11 Volgersi all'indietro per fare un bilancio di quanto accaduto, però, non significa voltare le spalle al futuro. Che lo abbiamo pensato o meno, i « cittadini di Arras », rivolgendosi nel modo che abbiamo riportato in esergo ad una Convenzione Nazionale appena liberatasi dal giogo del loro illustre conterraneo, non stavano facendo solo un resoconto comparativo dell'ultimo inatteso accadimento della Rivoluzione. La fine del Protettorato di Cromwell, infatti, aveva determinato qualcosa in più rispetto a quanto fino ad allora provocato dalla caduta di Robespierre: aveva favorito il ritorno della monarchia.

12 All'indomani del Termidoro, tuttavia, c'è un certo ottimismo sul futuro della Repubblica. Il passato sembra destinato a non dover tornare più. Ormai, i più lo usano in modo retrospettivo, per dimostrare come la storia recente sia stata una sfortunata riedizione delle grandi usurpazioni verificatesi nell'antichità e replicatesi a metà Seicento in Inghilterra $^{28}$. Due scenari esemplari che, combinati tra loro, consentono, al contempo, di denunciare i misfatti già commessi da Robespierre (nelle vesti di un Cromwell redivivo) ${ }^{29}$ e di svelare le sue ultime, velleitarie ambizioni (ispirate alle gesta di Catilina) ${ }^{30}$. L'immagine è quella di una repubblica che punisce il suo Protettore dopo aver sventato la sua ultima Congiura. Chi è sopravvissuto alla morte di Robespierre, in altre parole, vuole far credere che il peggio sia passato. L'Incorruttibile viene presentato come l'ennesimo Catilina smascherato per tempo o come un Cromwell incompiuto ${ }^{31}$. Ne è convinto Stanislas Louis Marie Fréron, tra i feroci esecutori del Terrore il più svelto a schierarsi dalla parte dei vincitori: "Plus heureuse que l'Angleterre, parce qu'elle avait plus de lumiers, parce qu'elle était plus digne de l'être, la France devait recevoir aussi cette derniers leçons, elle devait avoir un Cromwel, mais elle ne devait pas avoir un maître ${ }^{32}$.» I fatti gli daranno torto. Alla vigilia del primo anniversario del 9 Termidoro, mentre la pubblicistica è ancora impegnata a raccontare ai francesi i crimini del robespierrismo per interposta epoca storica ${ }^{33}$, l'attualità prende di nuovo il sopravvento. Saranno le insurrezioni di germinale e pratile (aprile e maggio 1795) a riaccendere la miccia della rivoluzione, rimettendo all'ordine del giorno la travagliata esperienza repubblicana inglese ed il suo antecedente romano ${ }^{34}$. Le coeve pubblicazioni in francese e la ricezione sulla stampa di opere a tema storico riguardanti autori e attori del repubblicanesimo inglese come James Harrington ${ }^{35}$, Algeron Sidney e Thomas Gordon ${ }^{36}$, non servono più solo a compilare bilanci, ma anche ad immaginare il futuro ${ }^{37}$. Il passato è ancora lo strumento privilegiato da chi ha il compito di ricostruire l'architettura istituzionale della Repubblica, preservandola dal rischio della decadenza ${ }^{38}$. A tal proposito, è emblematica questa frase del traduttore di Harrington inserita nell'introduzione alla versione francese (datata 1795) delle sue Opere politiche:

Les troubles de la Révolution française ont trop de ressemblance avec ceux de la Révolution d'Angleterre, pour que celui qui aime à remonter des effets aux causes, ne s'empresse pas d'étudier l'une, pour mieux deviner les suites de l'autre ${ }^{39}$

Del resto, basta scorrere il famoso discorso di François Antoine Boissy d'Anglas sul progetto di costituzione pronunciato il 5 messidoro anno III, per intuire in che misura la storia sia tornata ad essere insieme lo specchio del passato e del futuro della Rivoluzione. Sono trascorsi ormai sei anni esatti da quando il Terzo Stato si è ribellato a Luigi XVI (23 giugno 1789). Il re è morto. La Rivoluzione, invece, ha appena battuto l'ennesimo fragoroso colpo al grido «Pane e Costituzione del Novantatre». Lo sguardo rivolto all'indietro, Boissy d'Anglas vede solo un cumulo di macerie ${ }^{40}$. Non c'è più tempo da 
perdere, incalza il convenzionale. È giunto il momento di abbandonare definitivamente le secche della storia e di puntare dritti al futuro. Nel farlo, però, è indispensabile tenere a mente le lezioni offerte da un passato che ormai ospita anche un capitolo dedicato alla storia della Rivoluzione francese. Anch'essa, come le altre rivoluzioni, non dovrà più tornare:

Puisque nous voulons devancer l'avenir, enrichissons nous du passé. Nous avons devant nous l'histoire de plusieurs peuples; nous avons la nôtre: parcourons le vaste champ de notre révolution, déjà couvert de tant de ruines, qu'il semble partout nous offrir les traces et les ravages du temps [...] Nous avons consommé six siècles en six années. Que cette expérience si coûteuse ne soit pas perdue pour vous [...] C'est en vous rappelant le point d'où vous êtes partis, le chemin où vous avez été entrainés, la position dans laquelle vous êtes, que vous pourrez assigner vousmêmes le terme où vous voulez arriver ${ }^{41}$

Boissy d'Anglas è convinto che fino a quel momento, nonostante i suoi successi, la Francia abbia girato a vuoto. Come era già accaduto a Roma, infatti, anche la Repubblica francese, appena nata, si è piegata sotto il giogo dei « decemviri ». Cosicché, inesorabilmente, dopo la conquista della democrazia, anche a Parigi è venuto il tempo dell'anarchia e dei tiranni ${ }^{42}$. Il popolo francese, però, è stato in grado di imitare i romani anche nella buona sorte, poiché come allora è riuscito ad abbatterli ${ }^{43}$. Per fortuna, la ruota della storia anche stavolta ha continuato a girare fino alla riconquista della libertà. L'imperativo, adesso, è impedire che essa continui a farlo assecondando nuovamente il ritmo ciclico delle rivoluzioni di stato. I timori di Boissy d'Anglas, del resto, sono più che fondati. Qualche settimana prima, infatti, la Repubblica aveva oscillato di nuovo sul baratro dell'anarchia. In occasione delle insurrezioni di germinale e pratile, infatti, la folla inferocita aveva sbattuto davanti agli occhi dell'allora presidente della Convenzione Nazionale la testa mozzata del collega Féraud. La marcia retrograda degli ultimi invasori della Convenzione, fa notare Boissy, è emblematicamente iscritta nella loro richiesta di riesumare la Costituzione approvata dai Giacobini nel 1793. Nel farlo, essi si sono mostrati per quello che sono: nient'altro che un focolaio anarchico a cui avrebbe fatto seguito l'ennesima vampata della tirannide ${ }^{44}$. È la costituzione, sentenzia il convenzionale, l'unica forza capace di prevenire "le retour de toutes les tyrannies en détruisant l'anarchie», il solo strumento che impedirà alla Francia di rivivere nuovamente, in un lasso di tempo brevissimo « siècles d'erreurs ${ }^{45}$. »

Non è un caso se lo stesso Boissy d'Anglas e molti tra i nuovi Costituenti siano attratti da un celebre modello istituzionale di derivazione classica, rivisitato dai repubblicani inglesi e americani: la costituzione mista ${ }^{46}$. Un regime che si basa sulla mescolanza delle tre più note forme di governo (monarchia, aristocrazia e democrazia), immaginato come l'unico rimedio in grado garantire l'equilibrio tra i poteri e di bloccare il decadimento inarrestabile di una repubblica dalla democrazia all'anarchia, dall'anarchia all'aristocrazia, dall'aristocrazia all'oligarchia, dall'oligarchia alla monarchia e da quest'ultima (di nuovo) alla tirannia. Un rischio, quest'ultimo, che qualcun altro, all'opposto di Boissy, in tono altrettanto profetico, associa proprio all'adozione di un sistema bicamerale bilanciato ${ }^{47}$. È il fantasma dell'aristocrazia, anticamera certa per il ritorno della tirannia e del regime monarchico, che agita le «notti insonni » di un suo collega. Alexandre Deleyre, infatti, è atterrito dalla possibilità che una divisione ineguale della rappresentanza assembleare e il caos da essa provocato conducano tramite un détour anarchico all'usurpazione ad opera di un « terzo potere » esecutivo (il Direttorio): 
Ces deux pouvoirs d'un sénat ou d'un conseil d'anciens, et d'un conseil de proposants, appelleront le contre-poids d'un troisième pouvoir pour concilier les deux, et dans les chocs d'une trop forte et trop longue opposition, fixer la prépondérance de l'un sur l'autre. De là naîtrait infailliblement la nécessité d'une dictature, ou d'une autre magistrature qui, d'abord provisoire et temporaire, deviendrait insensiblement perpétuelle, ou ne serait remplacée que par la monarchie. Si vous n'y prenez garde, une troisieme constitution, fruit d'une troisieme révolution, amenera peut-être une quatrieme constitution par une quatrieme révolution [...] A Rome, une dissention naturelle entre deux ordres de citoyens ; et pour remede à l'anarchie, une dictature, porte de la tyrannie ${ }^{48}$.

Nelle sue previsioni, la divisione del Legislativo in due assemblee dotate di poteri diversi e concorrenti, ancorché ispirata e ammantata dall'autorità di precedenti antichi, anziché equilibrare il sistema istituzionale condannerebbe la Repubblica francese a patire la stessa sorte toccata al Commonwealth inglese: «Douze années de démocratie ne suffirent pas à Cromwel pour extirper les germes de l'aristocratie [...] la royauté fut ressuscitée avec les deux chambres ${ }^{49}$. "Stavolta, oltretutto, il rischio è che la Rivoluzione replicando il più classico dei cicli rivoluzionari ripeta anche sé stessa:

Encore un fois n'ayons pas perdu nos longues peines, et ne retournons pas à l'esclavage par le chemin de sang qui nous a conduits à la liberté. Mais pour nos reposer des tourmentes de ses éléments orageux, gardons-nous de prendre un écueil pour un port. La crainte de l'anarchie enfanta la constitution royale. La trahison de la monarchie durent amener la constitution populaire; et les proscriptions et la démagogie nous entraînent vers l'aristocratie. Ainsi toujours un tyrannie nous précipiterait dans une autre, comme si nous étions condamnés à les essayer toutes, et que le peuple, ou le genre humain, ne pût, on ne sût que changer de calamités et de servitude ${ }^{50}$

Ma torniamo al ragionamento di Boissy d'Anglas. L'esito che egli teme è più o meno lo stesso di Deleyre. A dividerli, tuttavia, ci sono sia gli strumenti prescelti per scongiurarlo, sia l'identificazione del passo falso che innescherà la decadenza della Repubblica. Infatti, mentre il secondo teme le conseguenze di una deriva aristocratica, il primo è convinto che l'irreparabile avverrà se « le peuple fait de mauvais choix; si ses flatteurs l'emportent encore [...] s'il se livre encore au démagogisme féroce et grossier.» A quel punto, conclude Boissy, ai fautori della libertà non resterà che scegliere una tra le identiche parti riservategli dal copione della storia: «Il ne vous reste plus à vous-mêmes qu'à choisir entre l'échafaud de Sidney, la ciguë de Socrate ou le glaive de Caton ${ }^{51}$.»

Gli eventi successivi dimostreranno l'insuccesso dell'idea espressa dai Costituenti dell'anno III di usare la storia per costruire un futuro migliore. Allora, però, nessuno attribuirà, come era accaduto all'indomani del Termidoro per il Terrore, la morte della Repubblica all'uso erroneo del passato. Nel corso dell'anno III, infatti, era tornato d'attualità l'antico dibattito sugli effetti nefasti provocati dall'abuso delle parole ${ }^{52}$ ed in molti avevano imputato il fallimento della Rivoluzione alla natura instabile del popolo e all'uso sconsiderato che era stato fatto del suo nome e della sua storia ${ }^{53}$. Gli errori del popolo, così come gli orrori della Rivoluzione, di cui esso è stato allo stesso tempo complice e vittima, agli occhi dei Termidoriani erano apparsi come il risultato di un duplice e spesso concomitante abuso, delle parole e della storia, di un doppio e tragico travisamento della realtà ${ }^{54}$. 


\section{Le passé qui ne passe pas : l'Angleterre sous le Directoire}

19 La Francia del dopo Robespierre vacilla ancora attorno al baratro di Clio ${ }^{55}$. Ben presto, tutti devono ammettere che la lama dell'ultima ghigliottina non ha tolto fiato all'oracolo della storia. Sono ancora una volta le città antiche e l'Inghilterra degli Stuart a ricordare ai francesi che la loro Repubblica, da un giorno all'altro, potrebbe cessare di esistere: conquistata la libertà, i romani hanno dovuto patire il decemvirato, le usurpazioni di Mario e Silla, il triumvirato, la dittatura di Cesare, e, infine, l'Impero; proclamato il Commonwealth, a loro volta, gli inglesi sono caduti vittima del Protettorato di Cromwell, per poi tornare sotto l'egida degli Stuart ${ }^{56}$. Per non parlare della possibilità che siano direttamente gli eventi della Rivoluzione a replicarsi nel suo prossimo futuro ${ }^{57}$. Eventualità, queste, che hanno spinto molti scrittori a stilare veri e propri manuali delle rivoluzioni, anche in formato vademecum ${ }^{58}$, in cui il ritorno della storia è adombrato o minacciato ${ }^{59}$. Non dobbiamo attendere, infatti, che il grande Chateaubriand riesca finalmente a pubblicare a Londra il suo Essai historique, politique et moral sur les révolutions anciennes et modernes, considérées dans leurs rapports avec la Révolution française (18 marzo $1797)^{60}$, in cui interpreta e condanna « quel presente già passato che è la Rivoluzione ${ }^{61}$ " come un'imitazione sconsiderata delle "rivoluzioni antiche e moderne» (tra cui ovviamente annovera anche quella inglese, considerata come un antecedente indispensabile per l'avvento della rivoluzione in Francia). Decline and fall of Roman Empire di Edward Gibbon, ad esempio, circola già da tempo in una duplice edizione francese, unitamente alle celeberrime Considérations sur les causes de la grandeur des Romains, et de leur décadence di Montesquieu ${ }^{62}$. Per non parlare dei libri a tema 'rivoluzionario' rielaborati o tradotti da François-Henri Turpin, tra cui l'Histoire des révolutions d'Angleterre (una continuazione dell'omonima opera del Père d'Orléans) ${ }^{63} \mathrm{e}$ l'Histoire du gouvernement des anciennes républiques, ou l'on découvre la cause de leur élévation et de leur dépérissement (una traduzione dall'inglese di Edward Wortley Montagu) ${ }^{64}$. Oppure della preziosa storia per immagini dell'abate Millot (Tableaux de l'Histoire romaine), pubblicata postuma nel 1796. È sempre in questo anno che il cittadino Bulard, già autore di un Tableau historique des républiques tant anciennes que modernes (1793), stampa la sua monumentale Histoire abrégée des Républiques anciennes et modernes, où l'on voit leur origine et leur établissement, et les causes de leur décadence et de leur ruine ${ }^{65}$. Un'opera in quattro tomi dedicata alla storia di alcune tra le più celebri repubbliche antiche (Atene, Sparta, Tebe, Cartagine, Roma) e moderne (Venezia, Genova, Lucca, San Marino, Ragusa, Olanda, Svizzera, Ginevra, Stati Uniti). Un racconto che, non a caso, si sofferma anche sulla Rivoluzione francese, ormai ricompresa a pieno titolo nel novero dei più tradizionali cicli rivoluzionari ${ }^{66}$.

Il passato, ammonisce Bulard, va studiato con attenzione ${ }^{67}$. Nella vita di una repubblica, ad un certo punto, accade che un "ambizioso » o un "generale » seduca il popolo per sottometterlo al suo volere. E, visto che questo passaggio si è avuto in Francia, prosegue Bulard, tutto lascia presagire che prossimamente potrebbe arrivare il tempo dell'anarchia. La ruota della rivoluzione, infatti, ora che la tirannia è stata abbattuta, pende verso l'estremo opposto : «Vous verrez paraître la licence, et avec elle la chute de la République ${ }^{68}$.»

21 Sempre nel 1796, precisamente il 24 settembre, François-Marie-Joseph Riou esprime in aula la stessa preoccupazione. Il futuro presidente del Consiglio dei Cinquecento, infatti, 
avanza il sospetto che i monarchici stiano lavorando proprio in tal senso. Egli è convinto che quest'ultimi vogliano trascinare la Francia nel caos allo scopo di riattivare il compimento della profezia inglese (rimasto in sospeso dalla caduta di Robespierre). Un nuovo ed indispensabile intermezzo anarchico capace di ripristinare la simbiosi tra le due rivoluzioni e traghettare la Francia verso la restaurazione. Un obiettivo, questo, precisa Riou, a cui per ragioni diverse ma convergenti, aspirano anche « les féroces partisans du code absurde de $1793 \%$ :

Et alors, qui eût rendu le despotisme même désirable, le Peuple Français, fatigué de tant des secousses; cherchant partout le repos et ne le trouvant nulle part, eût été conduit insensiblement par quelque Monk adroit et perfide, sous l'antique ombrage de la monarchie ${ }^{69}$

È «l'alliance monstreuse de l'anarchie et du royalisme», precisa un repubblicano di vecchia data come Jean de Bry. Dopo il tempo di Catilina e Cromwell, sta per suonare l'ora di Cesare e Monk ${ }^{70}$. Si tratta, invero, di timori tutt'altro che infondati. Una restaurazione alla Carlo II, infatti, è l'ultima spiaggia per uomini come Joseph de Maistre ${ }^{71}$.

Ad un anno esatto da questi discorsi, è il noto colpo di stato direttoriale del 18 Fruttidoro anno V (4 settembre 1797) a materializzare il fantasma della restaurazione nell'atto stesso di sventarla.

24 I fatti si sono svolti così. All'origine di tutto c'è la nomina, in occasione del rinnovo parziale dei Consigli tenutosi nell'aprile 1797, di un folto ed eterogeneo gruppo di oppositori al regime. Un risultato elettorale che incrina l'egemonia parlamentare dell'esecutivo ${ }^{72}$. Quando inizia a circolare voce che la maggioranza del Direttorio sta meditando l'annullamento delle elezioni, qualcuno grida alla proscrizione. I tre direttori repubblicani, Barras, La Révellière-Lépaux e Reubell, ben presto, emarginano il loro collega monarchico Barthélemy, che nel frattempo ha trovato la solidarietà del vecchio Carnot, dichiaratosi indisponibile ad appoggiare il colpo di mano. Per la stampa realista non ci sono dubbi, la Francia si sta incamminando verso un futuro romano. Il tempo del Direttorio e della Repubblica è finito. È giunta l'ora del triumvirato ${ }^{73}$.

25 A fine estate, un giornale in cui scrive anche il realista La Harpe pubblica addirittura il Plan de conspiration che il «directoire exécutif triumviral» avrebbe approntato per ribaltare il responso elettorale ${ }^{74}$. Contemporaneamente, il monarchico Richer de Serizy invita i francesi a considerare le stupefacenti analogie esistenti tra la Francia del 1797 e l'« époque de la décadence du Bas-Empire ${ }^{75}$.» Il Direttorio, avverte l'Accusatore Pubblico, sta giocando con il fuoco. Prima o poi scatenerà una guerra civile, abbandonando la Francia nella mani di orde di soldati mal controllate da ambiziosi generali che, alla fine, potrebbero anche riuscire ad impadronirsi personalmente del potere ${ }^{76}$. Gli occhi di Richer sono puntati su Bonaparte. Accusato non a torto di complicità con i triumviri, quest'ultimo gli appare tutt'altro che somigliante al tanto atteso Monk: «Non que je veuille à ces grands noms que je viens de citer assimiler celui de Bonaparte; non, je n'attends de cet homme que ruines, carnage et destruction Je n'attends de cet homme que ruines, carnage et destruction ${ }^{77}$. » Il timore è che le Alpi diventino il Rubicone della Repubblica francese, come si evince da questa dichiarazione del 19 agosto 1797 inoltrata a Parigi dagli amministratori dipartimentali del Rodano: «L'expérience de l'histoire nous apprend que si l'énergie des Peuples fonda la liberté, une armée qui marche et veut agir en son nom, ne peut que la détruire. Ce fut en combattant pour la liberté des Romains, que Jules-César parvint à les asservir ${ }^{78}$.» L'ombra di Cesare si aggira pericolosamente sulla testa del generale Bonaparte. Egli, del resto, non fa nulla per evitare le orme del dittatore romano. 
In quegli stessi giorni, come è noto, lavora per farsi mettere a capo di una spedizione militare in Egitto ${ }^{79}$. Gli uomini del Direttorio, tuttavia, si mostrano più fiduciosi: le misure predisposte dall'esecutivo in materia di destituzioni degli ufficiali militari consentiranno alla Repubblica di stroncare sul nascere qualsiasi emulo di Agatocle, Mario, Silla, Cesare e Cromwell $^{80}$. L'opposizione, però, insiste. L'avvocato di Maria Antonietta, Guillaume Alexandre Tronsson du Coudray, chiede ai Direttori: «Avez-vous relu les pages de l'histoire? avez-vous songé au prix qu'ont recueilli de tout tems les hommes qui ont fait ou laissé intervenir les armées dans leurs causes ${ }^{81}$ ? » Il tutto mentre all'uscita dai Consigli circola un pamphlet (attribuito al deputato filo-governativo Bailleul) che accusa la fazione realista di preparare il terreno all'avvento di Monk ${ }^{82}$.

Insomma, lo scontro politico avviatosi dopo le elezioni di aprile, a detta di molti, sembra essere destinato, in un modo o nell'altro, a decretare la fine dell'esperienza repubblicana per mano di un generale. La Francia del 1797 vive avvolta nelle nebbie del passato. Prima che arrivi l'autunno, però, ci pensa il Direttorio a fare chiarezza.

Nella notte tra 4 e 5 settembre 1797 (17-18 fruttidoro anno V), Barras, La RévellièreLépaux e Reubell, con il sostegno del generale Augereau, epurano i Consigli e ordinano la deportazione di molti deputati, compresi i Direttori dissenzienti Barthélemy e Carnot. Ben presto, le voci di La Harpe e Richer de Serizy saranno messe a tacere ${ }^{83}$. Non prima, però, di aver constatato l'avveramento delle loro infauste previsioni. Il tanto atteso ritorno di Cromwell è finalmente sotto gli occhi di tutti. L'anno seguente, anche l'abbé Duvoisin $^{84}$ e il noto giornalista Jacques Mallet du Pan certificano che il Direttorio è la nuova reincarnazione di Cromwell ${ }^{85}$.

Nel frattempo, mentre l'Accusateur public fa sapere che l'epurazione dei Consigli è stata una riedizione della purga del Lungo Parlamento eseguita dal generale Pride ${ }^{86}$, il giornale a cui collabora La Harpe, che nel frattempo ha assunto il nome di Tablettes historiques ${ }^{87}$, continua a paragonare gli ultimi risvolti della Rivoluzione alle antiche proscrizioni che hanno funestato la storia greca e romana ${ }^{88}$. Finché sulle Tablettes non compare il profilo di Ottaviano, ovvero di colui che, dopo aver preso parte ad un regime triumvirale, è riuscito a svuotare dall'interno le istituzioni repubblicane, inaugurando una nuova dinastia ${ }^{89}$.

Il destino della Francia sembra segnato: dopo il triumvirato di Barras e compagni, all'orizzonte si intravede l'approdo imperiale. Del resto, è nella logica dello schema comparativo inaugurato a luglio con la pubblicazione del Plan de la conspiration triumvirale, che dopo una dittatura collegiale venga il tempo dell'uomo forte ${ }^{90}$. Anzi, precisa Richer, i rivoluzionari francesi, con il manuale di storia alla mano, hanno addirittura saltato le tappe, catapultando la Francia dalla monarchia di Luigi XVI all'età del Basso Impero di Roma, senza nemmeno passare attraverso la sua stagione repubblicana ${ }^{91}$. E così, $i$ rivoluzionari si trovano nell'imbarazzante condizione di dover cedere il pugnale di Bruto nella mani del monarchico Richer de Serizy ${ }^{92}$, novello adepto di quel famoso Killing No Murder pubblicato per la prima volta nel 1657 per istigare al tirannicidio di Cromwell ${ }^{93}$.

30 Nel frattempo, però, i partigiani del secondo Direttorio hanno già archiviato la repressione di Fruttidoro come l'ennesima sconfitta di Catilina ${ }^{94}$. Benjamin Constant rassicura i francesi: il torrente della Rivoluzione non ha sfondato gli argini repubblicani. Fruttidoro non è una riedizione del 1648: « Ce n'est point Cromwell, cassant un parlement rebelle à ses volontés, c'est le génie de la République, repoussant du pouvoir des mandataires égarés ou infidèles ${ }^{95}$." Per il momento, accontentiamoci di sapere che Charles Bailleul ha finalmente dato un volto, anzi due, al suo Monk: è quello dei generali proscritti Pichegru e Willot ${ }^{96}$. Ma è solo l'inizio. Come previsto, infatti, l'anno seguente 
toccherà ai nostalgici dell'anno II, anch'essi smascherati per tempo dal Direttorio prima che facessero crollare l'edificio repubblicano.

31 Al momento opportuno, infatti, il 22 Floreale anno VI (11 maggio 1798), il governo fa scattare un altro colpo di stato per impedire l'ingresso nei Consigli di un cospicuo numero di deputati accusati di fomentare la conspiration anarchiste ${ }^{97}$. Al che, Lucien Bonaparte, il fratello di Napoleone, neo-eletto tra le fila dell'opposizione, avverte che se non si porrà un argine all'illegalità governativa dopo il triumvirato la Francia si ritroverà davanti ad un Cesare o ad un Cromwell:

Toutes les époques de l'histoire nous reproduisent les mêmes événemens sous d'autres noms. Cromwel, comme les triumvirs, voulait, disait-il, le perfectionnement de la constitution d'Angleterre [...] Le tyran descendit au sein du sénat; il ordonna à quelques soldats de dissoudre ce mannequin de représentation. Les soldats obéirent, et la révolution anglaise, qui avait coûté tant de sang, périt sous ce perfectionnement prétendu : et nous nous tairions! Et nous souffririons qu'on employât l'armée à la violation des droits des Peuples! ${ }^{98}$

Ed è proprio questo che accadrà un anno dopo. Peccato, però, che al momento della verità, il profeta Lucien offrirà all'aspirante dittatore il suo mantello per coprire la statua di Clio. Salvo poi giocargli un brutto scherzo. Quando Napoleone rimarrà solo davanti allo specchio della storia, infatti, sarà proprio il fratello a scoprirlo inaspettatamente.

\section{Simuler et dissimuler : Napoléon face au miroir anglaise}

Nel corso dell'anno VII, come di consuetudine, la Repubblica francese celebra i suoi anniversari. Stavolta, però, più che mai, le ricorrenze sono tutt'altro che scontate $\mathrm{e}$ retoriche. Il 1799, infatti, è l'anno in cui mentre si rimettono in scena simbolicamente le grandi giornate del passato, si fa strada il timore (o l'auspicio) che la storia rivoluzionaria possa replicarsi davvero e che possa farlo reiterando o portando a compimento l'identificazione con il suo doppio inglese ${ }^{99}$.

21 gennaio 1799. A sei anni dalla decapitazione di Luigi XVI, il peso della storia si fa sentire.

È come se la Francia fosse inchiodata al primo anno della sua era repubblicana e all'eventualità mai scongiurata di precipitare nel baratro di una restaurazione all'inglese:

Le supplice de Charles fut l'opprobre de l'Angleterre dont il commença l'esclavage; celui de Louis fut l'honneur de la France dont il assura la liberté [...] Jurons donc une haine éternelle à la royauté; jurons que cette haine s'étendra sur tous les genres de tyrannie; la royauté est la tyrannie d'un seul contre tous: cette lutte entraine la ruine de tous contre tous : cette lutte entraine la ruine de l'état social, et ramene à la tyrannie d'un seul; jurons haine éternelle à l'anarchie ${ }^{100}$

I francesi hanno compiuto un salto in avanti spettacolare, un viaggio mai sperimentato prima d'allora nella storia dell'umanità. Eppure, i superstiti del decennio rivoluzionario sono consapevoli di trovarsi ancora in bilico tra passato e futuro. L'anniversario del regicidio fa tornare alla mente tutti i fatti di una Rivoluzione interminabile ${ }^{101}$. Da qui il timore che prima o poi qualche sconsiderato riattivi la miccia, provocando il ritorno della monarchia attraverso l'intermezzo di una guerra civile.

Non a caso, è il repubblicano Algeron Sidney, oppositore di Cromwell e martire della restaurazione, l'eroe dell'allora direttore La Reveillère-Lépeaux ${ }^{102}$. Ancora una volta, però, la scelta ricade sull'idolo sbagliato. Di lì a poco, infatti, l'insuccesso del Direttorio alle 
elezioni primaverili dell'anno VII (aprile 1799) a vantaggio dell'opposizione repubblicana porterà alla rimozione dello stesso La Reveillère e dei suoi colleghi Merlin de Douai e Treilhard (16-18 giugno).

14 luglio 1799. Prima che cali il sipario, durante l'estate del Novantanove i francesi ricelebrano la presa della Bastiglia, la caduta di Robespierre e la dichiarazione della patria in pericolo. Nel 1789 la bella stagione era culminata sulla punta delle picche scortate dalla Guardia Nazionale di La Fayette alla volta di Versailles. Nel 1799, invece, si concluderà a Saint-Cloud, con la sfilata dei pretoriani di un altro e più temibile generale. Iniziata fuori Parigi, la Rivoluzione muore a cinque miglia dalla capitale. Sieyès, custode della memoria rivoluzionaria, ne sarà ancora una volta l'artefice. L'abate avrà, come pochi altri, il privilegio di rimettere in scena il suo stesso ruolo. Come sempre, però, egli non riuscirà a giocare a pieno la parte dell'oracolo della costituzione. Ancora una volta, le cose non andranno come previsto ${ }^{103}$.

Il 14 luglio sembra che tutti aspettino e temano il ritorno di qualcosa. In qualità di presidente del Direttorio, è l'abate Sieyès a presenziare la cerimonia. « Nous avons besoin d'une grande et républicaine énergie », dichiara solennemente, " reprenons, ressuscitons celle qui nous animait tous au 14 juillet." A patto, però, precisa il portavoce dell'esecutivo, di non lasciar uscire dal vaso della storia anche coloro che allora hanno usurpato la voce del popolo: « Français! Profitez des leçons d'une longue expérience; elles nous ont coûté assez cher ${ }^{104}$. » I fantasmi di Sieyès, in realtà, ad uno ad uno stanno tornando in vita: al Maneggio, infatti, è appena rinato il club dei Giacobini; nel corso di un contro-anniversario officiato da militari e deputati, inoltre, il generale Jourdan ha evocato l'altro volto della rivoluzione dell'Ottantanove, da sempre temuto e occultato, quello del popolo in armi: À la résurrection des picques !, è la sua parola d'ordine ${ }^{105}$. Ma questo passato non deve tornare, tuona al Consiglio dei Cinquencento il fratello di Napoleone, da qualche tempo in combutta con il presidente del Direttorio: «Point de convulsions, point de changemens de systême, point d'échafauds. » L'anarchia, ricorda, è l'anticamera della restaurazione. Per evitarla, per impedire che le rette parallele dei monarchici e dei nostalgici del Novantatre trascinino la repubblica «au milieu des convulsions de la guerre civile », intima Lucien, il Direttorio non dovrà più essere il « manichino » dei Consigli, né tantomeno dovrà trasformasi nuovamente in una specie di « triumvirato ${ }^{106}$. » Ma è quel che sarà.

9 Termidoro anno VII. Da un anniversario all'altro. A cinque anni dalla caduta di Robespierre, il presidente degli Anziani Louis Dubois Du Bais sollecita i deputati a "profiter des leçons d'une longue et trop malheureuse expérience pour en éviter le retour. » L'invito è a riunirsi attorno alla Costituzione dell'anno III per sconfiggere una volta per tutte $\mathrm{i}$ « due mostri » terribili della Repubblica: « le royalisme et le fanatisme ». Altrimenti, avverte il presidente, "nous rentrerons dans un chaos révolutionnaire inextricable »: «le peuple ne pouvant plus croire à la république, se jetterait, en nous maudissant, dans le bras de la royauté. » Un'eventualità resa ancora più probabile dal fatto che Cromwell si è già reincarnato in quel Robespierre di cui ricorre la caduta ${ }^{107}$.

Un "avvenire sinistro" aleggia sulle sorti dei repubblicani francesi, avverte Joseph Echasseriaux: «L'échafaud de Barnevelt et de Sidney, voilà le sort de tous les amis de la liberté dans tous les pays du monde ${ }^{108}$.» La restaurazione, fanno notare dal nuovo Direttorio, sta già andando in scena in Italia. Il pericolo è sempre più imminente, ora che Sidney è tornato a morire nelle repubbliche sorelle: 
Si les pages sanglantes de l'histoire d'Angleterre, du regne de Charles et de Jacques II, ne suffisent pas pour vous peindre le sort que destinent à la France ceux qui veulent y relever le trône, si les échafauds de Sydney ne sont pas assez éloquens, profitez de l'exemple que vous offrent à Milan et à Naples les tyrans qui se sont un moment ressaisis de la puissance ${ }^{109}$

41 Primo agosto 1799. Come al solito, i redattori del Moniteur hanno seguito con attenzione gli ultimi risvolti della cronaca politica. Oltre ai fatti di pratile, c'è molto altro da raccontare ai francesi. Stavolta, però, non si tratta di un colpo di stato o di una giornata rivoluzionaria. È di nuovo il tempo dei pamphlet. Sono tornati di moda gli oroscopi rivoluzionari. Il giornale ne mette a confronto tre, pubblicati tra maggio e luglio, a ridosso della crisi che ha portato all'epurazione dell'esecutivo: l'Essai sur les causes qui en 1649 amenèrent en Angleterre l'établissement de la République, sur celles qui en 1649 l'y consolider, sur celles qui l'y firent périr del deputato dei Cinquecento Boulay de la Meurthe; il saggio di Jean-Baptiste Salaville, l'antico collaboratore di Mirabeau, intitolato De la Révolution française comparée à celle de l'Angleterre, ou lettre au Répresentant du peuple Boulay (de la Meurthe), sur la différence de ces deux révolutions; l'opera di Benjamin Constant Des suites de la contre-révolution en Angleterre ${ }^{110}$.

La posta in gioco è altissima. Lo si capisce già dai titoli. Tutti vogliono tenere lontana la nave della Repubblica dallo scoglio inglese ${ }^{111}$. Un pesante interrogativo affligge i nostri autori: il ciclo rivoluzionario francese finirà come quello d'oltremanica, costringendo la Rivoluzione a tornare al punto di partenza? Boulay lo teme. Ed è per questo che il Moniteur si schiera dalla parte di Salaville, che dichiara: « Il n'y a aucune induction à tirer des progrès ni des suites qu'et la révolution en Angleterre, pour présager qu'elles seront les suites de la notre ${ }^{112}$. » Alle troppe analogie del primo, infatti, la redazione preferisce il più rassicurante parallelismo per contrasto dell'altro, che esclude in modo categorico la possibilità di una restaurazione all'inglese ${ }^{113}$. Così come apprezza l'idea di Constant di allestire il migliore tra gli scenari deterrenti allora a disposizione: un affresco impietoso della controrivoluzione, un "tableau effrayant des suites du rétablissement de la royauté en Angleterre $^{114}$. » Vale la pena segnalare come i punti di vista espressi da Salaville e Constant siano già contenuti in un'opera allora inedita di Madame de Staël, composta probabilmente tra maggio e ottobre dell'anno precedente ${ }^{115}$. Il manoscritto, intitolato Des circonstances actuelles qui peuvent terminer la Révolution et des principes qui doivent fonder la République en France, da un lato, attacca chi pensa che " le règne de Charles II a été doux ", dall'altro, ribadisce con forza " qu'il n'y a que des ressemblances apparentes entre la Révolution de France et celle d'Angleterre ${ }^{116}$. »

Ma torniamo alla cronaca. Al di là delle sue sincere intenzioni repubblicane, precisa il Moniteur, un inconveniente imbarazzante mina il Saggio di Boulay. Esso, infatti, avrebbe rinfocolato le speranze dei realisti, offrendogli un vademecum della controrivoluzione: «Il était dangereux de laisser aux espérances du royalisme un prétexte puisé dans la fausse supposition d'une identité parfaite entre les deux révolutions. » Ad ogni buon fine, però, pur in accordo con Salaville («ils on pu revenir sur le passé, nous ne le pouvons pas »), la redazione invita i francesi a tenere a mente anche gli avvertimenti di Boulay de la Meurthe. Non è per nulla escluso, infatti, che ancora una volta «les excès de la démagogie et de la tyrannie populaire" possano riattivare il ciclo rivoluzionario e favorire il ritorno della monarchia:

Quoique les independans en Angleterre aient eu un caractere diffèrent des factieux connus parmi nous sous le nom d'anarchistes [...] ils ont dans leurs rangs une foule 
de royalistes deguisés et d'agens de l'étrange qui les poussent à tous les extrêmes, et se servent d'eux comme d'un marchepied pour le rétablissement de la royauté ${ }^{117}$

È questa, infatti, la tesi di Boulay, che sembra meno preoccupato per un'eventuale ricomparsa di Cromwell, a suo dire già reincarnatosi in Robespierre, e più atterrito dal ritorno del radicalismo rivoluzionario ${ }^{118}$. Il suo ragionamento, espresso attraverso una " habile mise en scène du danger de la Restauration ${ }^{119}$ ", è coerente con la strategia messa in atto da Sieyès per destabilizzare l'equilibrio politico e ottenere una revisione dell'assetto istituzionale voluto dai Costituenti dell'anno III. L'obiettivo è delegittimare l'apparato istituzionale, evidenziando la sua incapacità di arginare la deriva anarchia (preludio della Restaurazione) alimentata dai nuovi Indipendenti ${ }^{120}$.

18 e 19 Brumaio anno VIII (9-10 novembre 1799). Gli Anziani, non tutti a dire il vero, convocati d'urgenza alle sette e trenta del mattino, decretano il trasferimento del Corpo legislativo e del Direttorio esecutivo al castello di Saint-Cloud: una congiura in atto nella capitale minaccerebbe l'incolumità delle autorità repubblicane ${ }^{121}$. All'istante, il generale Bonaparte, posto ai termini del medesimo decreto a capo delle truppe parigine, fa il suo ingresso nella sala delle Macchine. Ha un solo obiettivo: scacciare i fantasmi del passato. Quello che sta per fare insieme ai suoi "compagni d'arme " e sotto l'egida di Sieyès sconsiglia di tenere aperto il vaso di Clio. Qualche riflesso della storia potrebbe scuotere i rappresentati del popolo e accecare per sempre le sue ambizioni: «Qu'on ne cherche pas dans le passé des exemples qui pourraient retarder votre marche!». Anzi, visto che altrove (nella Repubblica batava del generale Daendels) è appena accaduto qualcosa di simile a quanto si appresta a realizzare ${ }^{122}$, Napoleone mette le mani avanti: « Rien dans la fin du 18 siecle ne ressemble au moment actuelle ${ }^{123}$. »

Non è facile, però, rimuovere dalla sua divisa la polvere della storia ${ }^{124}$. Le somiglianze sono già troppo forti per nascondersi dietro le apparenze. Tanto che alla fine sarà costretto a conviverci. Al momento, però, si va avanti, per imprudenza più che con dolo, con il gioco delle differenze.

I lettori del Moniteur lo scoprono leggendo il resoconto della giornata del 18 Brumaio. La redazione è venuta in possesso di uno scritto intitolato Dialogue entre un membre du conseil des anciens et un membre du conseil des cinq-cents. Si tratta di un testo distribuito "à neuf heures autour des conseils », subito dopo la comparsata in aula di Napoleone. Da un lato, la Cassardra dei Cinquecento, che intravede le ombre di Cesare e Cromwell dietro l'operazione in corso. Dall'altro, l'incredulo rappresentate degli Anziani, che rassicura i colleghi sul fatto che la profezia stavolta non si avvererà: « Un César, un Cromwel!...... Mouvais rôles, rôles usés, indignes d'un homme de sens, quand ils ne le seraient pas d'un homme de bien ». In caso, contrario, egli si dichiara pronto a tirare fuori il pugnale di Bruto ${ }^{125}$.

Il giorno seguente, alle porte dei Consigli, riuniti nella galleria dell'Apollon e nell'Orangerie del castello di Saint-Cloud, si presenta il tanto atteso e temuto generale. L'assemblea degli Anziani, nella sua maggioranza connivente con i congiuranti, ascolta senza troppi sussulti le parole del generale ${ }^{126}$. Bonaparte recita le stessa parte affidata al suo alter ego nel Dialogue:

On parle d'un nouveau César, d'un nouveau Cromwel; on répand que je veux établir un gouvernement militaire. Représentans du peuple, si j'avais voulu opprimer la liberté de mon pays, si j'avais voulu usurper l'autorité suprême, je ne me serais point rendu aux ordres que vous m'avez donnés, je n'aurais pas eu besoin de recevoir cette autorité du sénat. Plus d'une fois, et dans des circonstances extrêmement favorables, j'ai été appellé à la prendre ${ }^{127}$ 
49 È giunto di nuovo il momento di «salvare la patria » dal «pericolo » imminente di una « cospirazione ». Per chi conosce la storia romana (il senatus consultum ultimum e l'istituto del dictator seditionis sedandae causa) e ricorda i momenti di crisi e i dibattiti degli anni precedenti, si tratta di un déjà vu. Bonaparte chiede al « senato » i pieni poteri. Non certo la dittatura, anche se dalle sue parole sembra proprio: «Je vous déclare qu'aussitôt que les dangers qui m'ont fait confier des pouvoirs extraordinaires seront passé, j'abdiquerai ces pouvoirs. » Del resto, lo ammette, avrebbe potuto auto-investirsi già da tempo di un'autorità che, da ultimo, perfino i direttori Barras e Moulins gli hanno offerto. Ed è proprio a causa del suo rifiuto che ora lo denigrano come un Cesare o un Cromwell ${ }^{128}$. Adesso, però, ha ricevuto l'incarico di impedire che l'altro ramo del Corpo Legislativo riporti la Francia indietro, al tempo della "Convenzione» e della "ghigliottina». Bonaparte esce e va dai Cinquecento. Poco dopo, farà sapere agli Anziani di essere stato accolto dai loro indegni colleghi con $\mathrm{i}$ « pugnali ». Seguiamo il racconto cronologicamente sfalsato del Moniteur (il giornale, infatti, riporta come d'abitudine prima la seduta dei Cinquenceto e poi quella degli Anziani), che accredita questa leggenda. In apertura di seduta, un deputato vicino ai congiuranti dipinge la Repubblica sull'orlo dell'ennesima rivoluzione: «Jamais vous ne vites faire sous vos yeux plus de pas rétrogrades vers les formes monarchiques; jamais vous ne fûtes plus en butte ou aux suggestions royales, ou aux fureurs démagogiques. » Si capisce subito che non sarà una giornata facile. Ecco le reazioni della sala: «Plusieurs voix. Point de dictature, à bas les dictateurs [...] Une foule de membres se portent au bureau. Les cris à bas les dictateurs recommencent ${ }^{129}$. ${ }^{2}$ Colpo di scena. Eccolo, il dittatore. Bonaparte entra improvvisamente nella sala. Tra i banchi si scantena il putiferio: «Une foule de membres levés sur leurs siéges s'écrient : Hors la loi! hors la loi! A bas le dictateur!». Lucien, che in quel momento presiede la sessione, abbandona lo scranno e depone in segno di protesta «les marques de la magistrature populaire. » I granatieri lo scortano fuori dall'aula. Il pubblico salta fuori dalle finestre. I militari intimano ai deputati di ritirarsi. Il tumulto continua, finché quest'ultimi non abbandonano la sala al grido "vive la république ${ }^{130}$ ». Il resoconto finisce qui. Il Moniteur torna al punto di partenza, con una precisazione importante. Il presidente dei Cinquecento, nel frattempo, si è recato dagli Anziani per avvertirli che alcuni deputati " armés de pistolets et de poignards s'étaient portés sur le général »: "Bonaparte a été blessé au visage [...] et le granadier qui l'accompagnait a reçu le coup de poignard qui lui était destiné, et a eu la manche de son habit emportée ${ }^{131}$. »

L'uomo del destino è entrato nell'Orangerie preceduto da un'ombra che ha rischiato di schiacciarlo. Per sua fortuna, invece, sono stati i suoi antagonisti a soccombere. Tutto grazie al fratello. Con un gesto « alla romana », infatti, Lucien ha deposto le insegne della magistratura ed è uscito a cavallo nel cortile, «drappeggiato con la sua toga fiammeggiante ", per chiedere ai soldati di liberare immediatamente l'Assemblea dai « rappresentanti del pugnale ${ }^{132}$.» Qualche giorno dopo, Napoleone racconterà di un'aula assediata da uomini muniti di «stiletto e arma da fuoco ${ }^{133}$." In realtà, nessuno in quell'occasione ha trovato il coraggio di sfoderare il pugnale di Bruto ${ }^{134}$. Quest'immagine, insieme, menzognera ed eroica, che tradisce l'incapacità dei congiuranti di fare a meno (seppur a rovescio) della celebre sceneggiatura romana, passerà alla storia.

51 Come si vede, Napoleone oscilla tra la volontà di infrangere lo specchio della storia e la necessità di ricomporlo meticolosamente per presentare di volta in volta come legittimo un potere sospetto ${ }^{135}$. Prudenze e sfrontatezze imposte dall'incombere di un passato che non riuscirà mai a scrollarsi di dosso ${ }^{136}$. Chissà che faccia avrà fatto quando si è ritrovato 
tra le mani, per due volte di fila, nel 1800 e nel 1804, la versione francese del famoso Killing No Murder ${ }^{137}$. La prima volta, con questa premessa: «Si je suis un perfide, soyez tous des Brutus [...] Discours du général Bonaparte, au conseil des anciens, le 19 brumaire an 8 ${ }^{138}$.» L'altra, nell'anno dell'Impero, con questo titolo: Traduction du Pamphlet qui a fait mourir l'usurpateur Cromwell, intitulé: Tuer n'est pas assassiner. Dédié à Napoléon Buonaparte ${ }^{139}$. Il nostro racconto, iniziato con ricorrenza del regicidio, si conclude con un altro anniversario: la prima commemorazione del 18 Brumaio (9 novembre 1800). Nei giorni che precedono il ricordo della prima giornata, il momento in cui Napoleone ha iniziato la sua resistibile ascesa al potere, qualcuno fa circolare a Parigi e in provincia un insidioso e ambiguo pamphlet. Si tratta del famoso Parallèle entre César, Cromwel, Monck et Bonaparte: un testo anonimo di solito attribuito al monarchico Fontanes e politicamente addebitato nientemeno che a Lucien Bonaparte. È stato infatti l'apparato burocratico del Ministero dell'Interno, allora diretto dal fratello di Napoleone, a curare tra il 4 e il 6 novembre del 1800 la distribuzione capillare dello scritto in tutto il territorio della Repubblica, dopo averne assicurato la diffusione nella capitale ${ }^{140}$. Una manovra dai contorni oscuri, in cui pare che almeno inizialmente lo stesso Napoleone abbia avuto un ruolo ${ }^{141}$. Comunque siano andate le cose, si tratta di un bel pasticcio. A maggior ragione poiché il Parallèle esce a ridosso di una celebrazione importante, in cui Bonaparte avrebbe sancito simbolicamente la legittimità del suo potere ${ }^{142}$.

\title{
Conclusion : Les républiques après la République
}

\author{
« Le physique a un cours juste et régulier, ses \\ périodes sont fixés irrévocablement ; \\ le moral est régi par les mêmes lois ; \\ l'astronome connoit le cours des astres et annonce \\ leurs différens mouvemens. \\ Ainsi, le philosophe qui médite dans le silence et la \\ calme des passions, ne peut point se tromper \\ lorsqu'il prédit ses destinées » \\ Tableau historique et politique de la dissolution et du \\ rétablissement de la monarchie anglaise, \\ depuis 1625 jusqu'en 1702, par le citoyen J. Chas, Paris, \\ an VIII [1799], p. 268
}

Esempi come questi, ci inducono a prendere atto di come l'Ottantanove non abbia infranto affatto lo specchio della storia ${ }^{143}$. Ben oltre l'anno inaugurale della Rivoluzione, infatti, tutti gli attori in campo, senza distinzioni politiche ${ }^{144}$, hanno continuato a consultare il passato. La storia delle repubbliche (antiche e moderne) ha rappresntato un punto di riferimento ineludibile per i rivoluzionari francesi. Essa è stata una raccolta di soluzioni politiche da adattare all'attualità, un magazzino dove forgiare o ambientare idee e concetti con cui affrontare il presente e progettare il futuro. Del resto, è ormai risaputo che non esiste alcuna incompatibilità tra la volontà di cambiamento e la possibilità di pensarlo in analogia o per contrasto con eventi, protagonisti e istitutuzioni di un'altra epoca o di un'altra latitudine ${ }^{145}$. Oltretutto, è stato dimostrato come nel Settecento la visione lineare della storia non abbia soppiantato quella ciclica ${ }^{146}$. 


\section{NOTE}

1. Michel DE CERTEAU, L'Écriture de l'histoire, Paris 1975 ; Jacques RANCIERE, Les mots de l'histoire, Paris, Seuil, 1992 ; Francesco BENIGNO, Las palabras del tiempo. Un ideario para pensar históricamente, Madrid, Ediciones Cátedra, 2013.

2. Sull'uso della storia inglese durante la Rivoluzione si vedano almeno: Laurence L. BONGIE, David Hume: prophet of the counter-revolution (1965), Liberty Found, Indianapolis, 2000, Olivier LUTAUD, «Emprunts de la Révolution française à la première révolution anglaise. De Stuart à Capet, de Cromwell à Bonaparte ", in Revue d'histoire moderne et contemporaine, tome XXXVII, octobredécembre 1990, p. 589-607 e Roger BARNY, «L'image de Cromwell dans la Révolution française », in Dix-huitième siècle, 25, 1993.

3. Per la presenza delle reppubliche del passato nel discorso rivoluzionario, si vedano i contributi ricompresi in Michel vovelle (dir.), Révolution et République. L'exception française, Paris, Editions Kimé, 1994 e in Pierre SERNA (dir.), Républiques sœurs. Le Directoire et la Révolution atlantique, Rennes, Presses Universitaires de Rennes, 2009. Utile anche il saggio di Patrice GuENIfFEY, Cordiglieri e Girondini: la preistoria della repubblica?, in Furet, Ozouf (a cura di), L'idea repubblicana nell'Europa moderna, Laterza, Roma-Bari, 1993, p. 201-31.

4. Keith Michael BAKER, Inventing the French Revolution. Essays on French Political Culture in the Eighteenth Century, Cambridge, Cambridge University Press, 1990; François QUASTANA, La pensée politique de Mirabeau (1771-1789) : « républicanisme classique » et régénération de la monarchie, Aix-enProvence, PUAM, 2007.

5. Talbot Harold PARKER, The Cult of Antiquity and the French Revolutionaries. A Study in the Development of the Revolutionary Spirit, Chicago, The University Press of Chicago, 1937, p. 103 e ss.; Rachel HAMMERSLEY, French Revolutionaries and English Republicans. The Cordeliers club, 1790-1794, Woodbridge, The Boydell Press, 2005; Raymonde MONNIER, Républicanisme, patriotisme et Révolution française, Paris, L'Harmattan, 2005. Sull'uso della storia antica, inoltre, vista la quantità notevole dei contributi esistenti, rinviamo alla messa a punto teorica di François HARTOG, op. cit., e al caso di studio preso in esame da Luca SCUCCIMARRA ( Sorvegliare e punire. Rivoluzione francese e istituzioni di controllo », in Il Pensiero Politico, 2, 2007, p. 434-462), da cui emergono con chiarezza le ragioni che hanno spinto i rivoluzionari a riesumare le magistrature di controllo attive nelle repubbliche classiche al fine di riformare la monarchia costituzionale e poi di sconguirare la caduta della Repubblica.

6. "Mais si la rhétorique et le caractère du républicanisme ont été bien étudiés dans le contexte de la révolution anglaise et, pour le $18^{\mathrm{e}}$ siècle, dans celui de la révolution américaine, ce n'est pas le cas pour la France, où la recherche ne fait que commencer »: Raymonde MONNIER, op. cit., p. 38.

7. Annie JOURDAN, La Révolution, une exception française?, Paris, Flammarion, 2004.

8. Pierre SERNA, Le Directoire, miroir de quelle République?, in Id. (dir.), op. cit., p. 7-20; Manuela ALBERTONE, Antonino DE FRANCESCo (ed. by), Rethinking Atlantic Word. Europe and America in the Age of Democratic Revolutions, New York, Palgrave Macmillan, 2009; Raymonde MONNIER, "Tradition et innovation: transfert et réception des textes républicains autour de 1789 ", in Révolution Française.net, juillet 2011(http://revolution-francaise.net/2011/07/07/444-tradition-etinnovation-transfert-et-reception-des-textes republicains autour-de-1789).

9. Mentre Mona ozouf, Varennes. La mort de la royauté, Paris, Gallimard, 2005; Andrew JAINCHILL, Reimagining Politics after the Terror: the Republican Origins of French Liberalism, Itacha, London, Cornell University Press, 2008, p. 22. 
10. Per una ricognizione critica di tali studi si veda Marco GEUNA, « La tradizione repubblicana e i suoi interpreti: famiglie teoriche e discontinuità concettuali », in Filosofia politica, 1, 1998, p. 102-132.

11. Rachel HAMMERSLEY, op. cit., p. 2-7.

12. Jacques DE SAINT-VICTOR, Les racines de la liberté. Le débat français oublié 1689-1789, Paris, Perrin, 2007, p. 247-248.

13. Keith Michael BAKER, "Transformations of Classical Republicanism in Eighteenth-Century France ", in The Journal of Modern History, 73, 2001, p. 32-53; Mona ozouf, op. cit., p. 249.

14. James LIVESEY, Making democracy in the French Revolution, Cambridge, 2001.

15. «Thermidorian classical republicanism, I argue, signaled a return to a more traditional form of classical republicanism. It was antidemocratic and elitist, restricting the franchise and looking to the virtuous legislator rather than the virtuous people as the foundation of the nation's political order »: Andrew JAINCHILL, "The Constitution of the Year III and the Persistence of Classical Republicanism», in French Historical Studies, 26, 3, 2003, p. 401. Una tesi criticata da Antonino De Francesco, Traduzioni e Rivoluzione. La storia meravigliosa della prima versione in francese del Federalist (Paris, Buisson 1792), in « Rivista storica italiana », 2011, I, p. 100-1.

16. Andrew JAINCHILL, art. cit., p. 433-434.

17. " There was, however, one group of revolutionaries - associated with the radical, Paris-based Cordeliers Club - that made extensive, and surprisingly consistent, use of a substantial number of English republican texts during the French Revolution. They did so in order to develop, support and justify their own distinctive version of French revolutionary republicanism. This republicanism, which they developed in opposition to the modern republicanism of the Brissotins, was extremely democratic in nature »: Rachel HAMMERSLEY, The English republican tradition and eighteenth-century France. Between the ancients and the moderns, Manchester and New York, Manchester University Press, 2010, p. 14.

18. Chryssanthi AVlami, La Grèce dans l'imaginaire libéral ou, comment se débarasser de la Terreur e François HARTOG, La Révolution française et l'Antiquité. Avenir d'une illusion ou cheminement d'un quiproquo?, in Chryssanthi AVLAMI (dir.), L'Antiquité grecque au XIXème siècle. Un exemplum contesté? , Paris, L'Harmattan, 2000.

19. John G. A. POCOCK, «Cittadini, clienti e creditori: la repubblica come critica del mutamento storico ", in Maurizio VIROLI (a cura di), Libertà politica e virtù civile. Significati e percorsi del repubblicanesimo classico, Torino, Fondazione Giovanni Agnelli, 2004, p. 133, nota 1.

20. «Le lien établi avec les textes de la tradition radicale anglaise replace les théories démocratiques de la révolution française dans le champ des révolutions de la fin du $18^{\mathrm{e}}$ siècle et dans la trace séculaire des théories républicaines modernes de la liberté »: Raymonde MONNIER, op. cit., p. 14.

21. Ispirandosi alla riflessione di Baker, con riferimento ai Costituente dell'anno III Andrew Jainchill ha giustamente affermato: "They understood the problems facing the French Revolution in classical-republican terms and articulated solutions drawn from the classicalrepublican repertoire " (Andrew JAINCHILL, art. cit., p. 399). Non è fondata, tuttavia, la tesi di Jainchill circa una differenza sostanziale tra il discorso sull'antichità pre e post Termidoro (Id., op. cit., p. 8-11 e 17-8).

22. Michael SONENSCHER, Before the Deluge: Public Debt, Inequality, and the Intellectual Origins of the French Revolution, Princeton University Press, Princeton, 2007; Keith Michael BAKER, «Enlightenment Idioms, Old Regime Discourses, and Revolutionary Improvisation », in Thomas E. KAISER, Dale K. VAN KLEY (edited by), From Deficit to the Deluge. The Origins of the French Revolution, Stanford, Stanford University Press, 2011, p. 177; Andrew JAINCHILL, op. cit., p. 6.

23. Laurence L. BONGIE, op. cit. 
24. Keith Michael BAKER, art. cit; Pierre VIDAL-NAQUET, Tradition de la démocratie grecque, Introduction à Moses FINLEY, Démocratie antique et démocratie moderne, Paris, Payot, 1976, p. 18.

25. Pierre SERNA, «1799, le retour du refoulé ou l'histoire de la Révolution anglaise à l'ordre du jour de la crise du Directoire ", in Philippe BOURDIN (dir.), La Révolution, 1789-1871. Écriture d'une histoire immédiate, Vizille, Presses Universitaires Blaise-Pascal, 2008, p. 219.

26. Sul Termidoro si veda Sergio LUZZATTO, L'autunno della Rivoluzione. Lotta e cultura politica nella Francia del Termidoro, Torino, Einaudi, 1994.

27. Moniteur, XI, n. 308, Supplément à la Gazette Nationale, 8 thermidor an II (26 juillet 1794), Librairie: Sallustio è tradotto da Brosses e Gibbon da André-Samuel-Michel Cantwell.

28. Sull'associazione immediata tra Robespierre e gli usurpatori del passato si veda Bronislaw BACZKO, op. cit., p. 54.

29. Si veda, ad esempio, la requisitoria contro Robespierre e il Terrore e il parallelismo con Cromwell di Edme Petit: Moniteur, XI, n. 360, Convention nationale, séance du 28 fructidor an II (14/09/1794), p. 1478-1479.

30. Galart DE MONTOJE, Histoire de la conjuration de Maximilien Robespierre, Paris, 1795.

31. Moniteur, XI, n. 323, tridi 23 Thermidor an II (10-08-1794), Convention Nationale, suite à la séance du 21 thermidor, p. 1322: «L'orateur de la députation. Représentans, le jour où dans cette enceinte vous arrachiez le masque aux Catilina modernes; le jour où, secondés du Peuple, vous exterminiez ces singes féroces et hypocrites des César et des Cromwell... ». Si veda, ad esempio, il Véritable portrait de Catilina Robespierre, tiré d'après nature di Jean-Joseph Dussault.

32. Moniteur, XI, n. 342, Convention nationale, suite à la séance du 9 fructidor an II, p. 1401.

33. "L'histoire de cet homme extraordinaire ne pouvait être publiée dans des circonstances plus favorables. Plusieurs événemens de son regne présentaient des comparaisons, des rapprochemens avec les événemens qui se sont passée dans le cours de notre révolution, et l'auteur les a saisis. C'est dans la conduite de ce tyran que nos derniers oppressuer avaient puisé les moyens de nous ramener à l'esclavage [...] On croira parcourir l'histoire du tems présent: les ressemblances mêmes sont si frappantes »: da una recensione del "Moniteur» XII, n. 222, 21/05/1795 a Antoine Jeudy Dugour, Histoire d'Olivier Cromwel, Paris, l'an $3^{\text {ème }}$.

34. Se si legge la recensione del Mercure français (n. 55, 5 messidor l'an troisième [23/06/1795], Littérature ancienne, Histoire) alla Conjuration de Catilina contre la république romaine, par Salluste, nouvellement traduite [...] avec un discours préliminaire et des notes littéraires et politiques, par J. B. L.J. Billecocq, Paris, an III, si capisce bene come agli occhi dei contemporanei la sceneggiatura romana evocava più i fatti di germinale e pratile che il Giacobinismo.

35. « Au lendemain de Thermidor, les idées d'Harrington jouèrent un rôle important dans le débat constitutionnel français. Elles étaient invoquées, tant pour supporter que pour s'opposer à la Constitution de 1795, et le furent de nouveau lors de l'élaboration de la Constitution de 1799 » (Rachel HAMmERSLEY, op. cit., p. 217). Ma su Harrington nella Rivoluzione si veda anche Ead., «The Commonwealth of Oceana de James Harrington: un modèle pour la France révolutionnaire ?», in Annales historiques de la Révolution française, n. 342, 2005, p. 3-20.

36. Rachel HAMMERSLEY, op. cit., p. 207-209.

37. Rachel hAMmERSLEY, «La France contre l'Angleterre, tout contre, ou lire les texts des républicains anglais au temps du Directoire », in Pierre SERNA (dir.), op. cit., p. 205-218.

38. "The disabused politicians of the Year III sounded classical-republican notes in emphasizing history as a repository of experience from which they could draw lessons to aid in the navigation of republican political life » (Andrew JAINCHILL, art. cit., p. 410).

39. Pierre F. HENRI, Préface du traducteur, in CEuvres Politiques de Jacques Harrington, Paris, an III (1795), p. I. Ma sulla rivalutazione di Harrington da parte di Henri, si vedano i lavori già citati Hammersley, tra cui da ultimo The English republican tradition and eighteenth-century France. 
Between the ancients and the moderns, Manchester University Press, Manchester and New York, 2010, p. 160-1.

40. Boissy D’ANGLAS, Discours préliminaires au projet de Constitution pour la République française, prononcé par Boissy-D'Anglas, au nom de la Commission des Onze, dans la séance du 5 messidor, an 3, p. 9. Sulla visione del tempo in Boissy, vedi Edouard Pommier, Boissy d'Anglas : culture et conscience de l'histoire en l'an II, in L'espace et le temps reconstruits. La Révolution française, une Révolution des mentalités et des cultures?, Publication de l'Université de Provence, Aix-en-Provence, 1990, p. 153-67 e anche Andrew JAINCHILL, art. cit., p. 409-12.

41. Boissy D'ANGLAS, op. cit., p. 7.

42. Cfr. Marc DELEPLACE, Comment sortir de l'anarchie ? Un paradoxe républicain en l'an III, in Marc BELISSA, Yannick BOSC, Florence GAUTHIER (dir.), Républicanisme et droit naturel. Des humanistes aux Révolution des droits de l'homme et du citoyen, Editions Kimé, Paris, 2009 p. 87-100

43. Boissy D'ANGLAS, op. cit., p. 4-5.

44. Ibidem, p. 21-29.

45. Ibidem, p. 17-8.

46. «In reformulating France's constitutional architecture, the commission turned to the classical-republican solution of order through balance [...] The intellectual genealogy of putting the three classical forms in a state of balance or equilibrium runs through the canonical figures of the classical republican tradition-from Polybius through Machiavelli, Harrington, Algernon Sidney, and Montesquieu to John Adams »: Andrew JAINCHILL, art. cit., p. 412-413. Si veda anche Rachel HAMMERSLEY, in Pierre SERNA (dir.), op. cit., p. 209-218. Sui riferimenti alla storia e alla momenclatura delle istituzioni repubblicane (classiche) in vista dell'introduzione di un "pouvoir régulateur " dopo il Termidoro, si veda anche Marcel GAUCHET, La Révolution des pouvoirs. La souveraineté, le people et la representation, 1789-1799, Paris, Gallimard, 1995, p. 125 e ss.

47. "Je l'avoue, un pressentiment de nouveaux malheurs, sorte d'éclair qui nous guide quelquefois dans les ténebres de l'avenir »: Moniteur, XIII, n. 305, 23/07/1795, Convention nationale, séance du 30 messidor an III, p. 1228. L'intervento è di Alexandre DELEYRE.

48. Ibidem.

49. Ibidem.

50. Ibidem, p. 1229.

51. Boissy D'ANGLAS, op. cit., p. 94.

52. Raymonde MONNIER, "Autour des usages d'un nom indistinct : "peuple » sous la Révolution française ", in Dix-Huitième Siècle, 34, 2002, p. 389-418; Jacques GuILHAumou, La langue politique et la Révolution française, Meridiens Klincksieck, Paris 1989, in part. p. 51-80; Ulrich RICKEN, « Réflexions du XVIIIe siècle sur "l'abus des mots" ", in Mots, 4, 1982, p. 29-37; Roger BARNY, « Les mots et les choses chez les hommes de la Révolution française ", in La Pensée, 202, 1978, p. 96-115.

53. Si veda, in particolare, il testo intitolato Considérations sur les abus des mots et des choses dans la révolution, et sur les principes qui peuvent la terminer, pubblicato a puntate dal Mercure nell'autunno del 1794 (nn. 6, 10, 13 e 16), che Sophia ROSENFELD (A Revolution in Language. The problem of Signs in Late Eighteenth-Century France, Stanford University Press, Stanford, 2001, p. 313) attribuisce a J.-J. Lenoir-Laroche.

54. Sull'intreccio tra l'abuso delle parole e della storia nell'anno III mi permetto di rinviare al mio "Abuso delle parole (e della storia) nella Rivoluzione francese: il popolo dell'anno III », in Luca sCUCCIMARRA, Giovanni RUocco (a cura di), Il governo del popolo. Dall'antico regime alla Rivoluzione, I, Roma, Viella, 2011, p. 331-355.

55. Sulla Francia del Direttorio, si rinvia almeno a Pierre SERNA, La République des girouettes ( 1789-1815... et au-delà). Une anomalie politique : la France de l'extrême centre, Seyssel, Champ Vallon, 2005 e Philippe BOURDIN, Bernard GAINOT (textes réunis par), La République directoriale, 2 vols, Clermont-Ferrand, Société des études robespierristes, 1998. 
56. Come si evince anche dall'Essai sur la vie T. Wentworth, comte de Strafford pubblicato all'inizio del 1795 da Lally-Tollendal.

57. Mona ozouf, "De thermidor à brumaire : le discours de la Révolution sur elle-même ", in Revue historique, 493, Janvier-Mars 1970, p. 31-66; Ead., " Thermidor ou le travail de l'oublie », in Ead., L'école de la France. Essais sur la Révolution, l'utopie et l'enseignement, Paris, Gallimard, 1984, p. 91-108; Ead., "Le décrets des deux-tiers ou les leçons de l'Histoire », in Roger DuPUY, Marcel MORABITo (dir.), 1795, pour une République sans Révolution, Rennes, Presses Universitaires de Rennes, 1996, p. 193-209; Alphonse AULARD, Études et leçons sur la Révolution Française, vol. 6, Les premiers historiens de la Révolution française, Paris, Alcan, 1910; J.-R. SURATTEAU, « L'Histoire de la Révolution par elle-même ", in La storia della storiografia europea sulla Rivoluzione francese, II, Roma, Istituto Storico italiano per l'età moderna e contemporanea, 1990, p. 7-16; Philippe BOURDIN (dir.), op. cit. Sulla memoria della Rivoluzione durante l'età del Direttorio si veda anche il caso Prudhomme, così bene esaminato da Joseph ZIZEK, «'Plum de fer': Louis-Marie Prudhomme Writes the French Revolution ", in French Historical Studies, vol. 26, n. 4 (fall 2003), p. 619-660.

58. Tablettes chronologiques des révolutions de l'Europe, du citoyent Koch, Membre de l'institut national, Strasbourg, an VI de la République [1798].

59. Si vedano, ad esempio, Emmanuel François DE TOULONGEON, Manuel révolutionnaire, ou pensées morales sur l'état politique des Peuples en révolution, Paris, l'an IV e Agathocles et Monk, ou l'Art d'abattre et de relever les trones, par M. Philippon, prêtre instituteur à Orléans, A Orléans, A Paris, Cinquième année républicaine.

60. Si tratta di un'opera avviata probabilmente nel 1793, parzialmente edita a Londra nel 1796 e diffusa in Francia solo nel Novantasette (Pierre CHRISTOPHOROv, Sur le pas de Chateaubriand en exil, 1961, p. 82-83). Su Chateaubriand si vedano François HARTOG, Régimes d'historicité: présentisme et expériences du temps, Seuil, Paris, 2003, p. 104-133 e Chryssanthi AVLAMI, L'antiquité grecque à la française: modes d'appropriation de la Grèce au XIX ${ }^{e}$ siècle, 2 vols., Thèse de Doctorat présentée à Paris le 13 janvier 1998, sous la direction de M. François Hartog, Ecoles des Hautes Etudes en Sciences Sociales, vol I, Les Grecs contre la Révolution : François-René de Chateaubriand, p. 14-98.

61. Nicole LORAUX, Pierre VIDAL-NAQUET, La formazione dell'Atene borghese. Saggio di storia della storiografia 1750-1850, in VIDAL-NAQUET, La democrazia greca nell'immaginario dei moderni, Milano, Il Saggiatore, 1996, p. 183.

62. Tali opere sono prontamente recensite sulla stampa: Moniteur, XII, $n^{\circ} 262$, [10/06/1795], p. 1058; Mercure Français, n. 24, décadi 10 pluviose l'an troisième, 29 janvier 1795. Sempre sul Mercure si ragiona attorno all'opera dell'abbé René Aubert DE VERTOT intitolata Histoire des révolutions arrivées dans le gouvernement de la République romaine (n. 19, décadi 10 germinal, l'an cinquieme, jeudi 30 mars 1797, Annonces, p. 104) e si segnalano opere simili come l'Histoire de la décadence des moeurs chez les Romains, et de ses effets dans les derniers tems de la république, traduite de l'allemand de C. Meiners, par René Binet... l'an $3^{\circ}$ de la république (n. 10, Décadi 20 brumaire, l'an quatrieme, mercredi 11 novembre 1795, p. 291-294).

63. Histoire des révolutions d'Angleterre, depuis le commencement de la monarchie jusqu'en 1747. Par le P. d'Orléans. Continuée par H. F. Turpin. Tome cinquième, Paris, an IV [1795].

64. Mercure Français, n. 37, décadi 10 messidor l'an quatrieme [28 juin 1796], Livres français, p. 36; Moniteur, XIV, n. 131, 31/01/1796, Livres divers, p. 524. Il Moniteur (XIII , n. 296, 15/07/1795, Livres divers, p. 1194) segnala tra le CEuvres divers de Mirabeau anche la traduzione dell'Histoire de l'Angleterre di Macaulay.

65. Segnalata dal Moniteur, XV, n. 353, [9/09/1796], p. 1412 e dal « Mercure Français », n. 1, décadi 10 vendémiaire, l'an cinquième (samedi 1 octobre 1796), p. 53.

66. BULARD, Histoire abrégée des Républiques anciennes et modernes, où l'on voit leur origine et leur établissement, et les causes de leur décadence et de leur ruine, tome IV, Caillot, Paris, 1795, p. 214-88.

67. Ibidem, tome I, Préface, p. 5. 
68. Ibidem, p. 12-8.

69. Moniteur, XV, n. 7, [28/9/1796], Conseil de Cinq Cents, séance du 3 vendémiaire an V, Riou, au nom d'une commission spéciale, chargée d'examiner la loi du 3 brumaire, et les discours prononcés contr'elle dans les stance des 22 et 23 fructidor an 4, p. 25-27.

70. Moniteur, XV, n. 30, [22/10/1796], Conseil des Cinq-Cents, séance du 25 vendemiaire an V, p. 119-120.

71. Considérations sur la France. Seconde édition, revue par l'Auteur, Londres, mars 1797. Su De Maistre vedi anche Laurence BONGIE, Waiting for General Monk, in Id., op. cit., p. 187-89 e Geoffrey CUBITT, «Revolution, Reaction, Restoration », art. cit., p. 28. Come è stato notato, i monarchici attendevano « un Cromwell, si l'on peut dire, 'post-mortem', c'est-à-dire à un Monk, facilitant sans heurt le retour dynastique »: Olivier LUTAUD, "Des Révolutions d'Angleterre à la Révolution Française ", art. cit., p. 241. Sull'evocazione di Monk da Fruttidoro a Brumaio, si veda ancora Laurence BONGIE, Waiting for general Monk, op. cit., p. 186-196.

72. Su questo periodo della Rivoluzione, si veda ad esempio Georges LEFEBVRE, La France sous le Directoire, 1795-1799, Paris, Tarrains, 1984, p. 405-456. Per una prima analisi del coupe di Fruttidoro e, in generale, su tutti i colpi di stato del Direttorio, rinviamo alla messa a punto di Luca SCUCCIMARRA, La sciabola di Sieyès. Le giornate di brumaio e la genesi del regime bonapartista, Il Mulino, Bologna, 2002, p. 97-141.

73. Le Mémorial, I, n. 62, 20/7/1797 (2 thermidor an V), Le Triumvirat, par un député, p. 2.

74. Le Mémorial, I, n. 64, 22/7/1797, (4 thermidor an V), Plan de la nouvelle conspiration triumvirale, p. 2 e n. 70, 28/7/1797, (10 thermidor an V), Fin du plan de conspiration triumvirale, p. 3.

75. L'Accusateur public, nn. XXXIII-XXXIV (7 Août 1797, 20 thermidor), p. 3-4.

76. Ibidem, p. 9-17.

77. Ibidem, p. 4-5.

78. Moniteur, XVI, n. 332, [19/08/1797], Lettre de l'administration départementale du Rhone, Lyon, le 22 thermidor, p. 1328.

79. Jean TULARD, Napoléon ou le mythe du sauveur, Paris, Fayard, 1992, p. 35.

80. Moniteur, XVII, n. 337, [24/08/1797], Conseil des Cinq-Cent, suite de la séace du 3 fructidor an V, p. 1348. L'intervento è di Pierre-Jean AUDOUIN.

81. Moniteur, XVII, n. 337, [24/08/1797], Conseil des Cinq-Cent, suite de la séace du 3 fructidor an V, suite du discours de Tronson-Ducourdray, p. 1348.

82. Moniteur, XVII, n. 349, [5/9/1797], Conseil des Cinq-Cents, suite de la séance du 13 fructidor an V, p. 1395.

83. Su Richer si veda Sergio LUZZATTo, L'autunno della Rivoluzione cit., p. 133-139.

84. Défense de l'ordre social contre les principes de la Révolution Française, Londres, 1798. Cfr. Lutaud, Des Révolutions d'Angleterre à la Révolution Française cit., p. 244.

85. "Mercure Britannique ou notices historiques et critiques sur les affaires du tems ", par J. Mallet du Pan, vol. II, Londres, n. IX, 25 Décembre 1798, p. 23.

86. L'Accusateur public, n. XXXV, p. 7.

87. Tale giornale, alla cui redazione collaboravano anche Fontanes e Vauxcelles, cambia ulteriormente titolo, prima di chiudere i battenti, sul finire del 1797, assumendo sarcasticamente il nome di Tablettes républicaines.

88. Tablettes historiques, I, n. 15, 21/11/1797 (1 frimaire an VI), Variétés. Des proscriptions, p. 122-123, n. VII, 28/9/1797 (7 vendémiaire an VI), Variétes. De l'ostracisme et de la déportation, p. 26-27, n. 28, 19/10/1797 (28 vendémiaire an VI), Variétés, Sur le projet de résolution, du 25 de ce mois, concernant l'expulsion des ci-devant nobles, p. 111.

89. Tablettes historiques, I, n. 18, 9/10/1797 (18 vendémiaire an VI), Variétés. D’Octave, p. 70-71.

90. Tablettes historiques, I, n. 9, 30/9/1797 (9 vendémiaire an VI), Variétés. Moyen de lever les difficultés qui peuvent diviser les deux premières autorités de l'Etat, p. 34. 
91. L'Accusateur public, n. XXXV, l'an VII, 1 frimaire, p. 38-41.

92. Ibidem, p. 86-89.

93. Ibidem, p. 12-13.

94. Moniteur, XVII, n. 354, [11/09/1797], Conseil des anciens, suite de la séance permanente du 19 an V, p. 1424. L'intervento è di Bordas.

95. Citato da Olivier LUTAUD, Des Révolutions d'Angleterre à la Révolution Française, art. cit., p. 244.

96. Moniteur, XVIII, n. 183, [23/3/1798], Corps législatif, suite du rapport fait, dans la séance du 26 ventose an $V$, Sur la conjuration du 18 fructidor, par J. CH. Bailleul, p. 735.

97. Ibidem, p. 483.

98. Moniteur, XIX, n. 334, [21/8/1798], Conseil des Cinq-cents séance du 3 fructidor an VI, p. 1340.

99. «Dans tous les cas chacun eut conscience que rarement l'histoire ne fut autant sur le point de se répéter et il se vint jamais à l'esprit de personne que cela pût être sous la forme d'une comédie ou d'une mascarade »: Pierre SERNA, art. cit., in Philippe BOURDIN (dir.), op. cit., p. 221.

100. Moniteur, XX, n. 126, [25/1/1799], Conseil des anciens, séance du 2 pluviose an VII, p. 514. A parlare è il presidente degli Anziani Garat, il cui intervento è riassunto dalla redazione del Moniteur. Sulla persistenza del regicidio nell'immaginario repubblicano, si vedano le considerazioni di Pierre SERNA, art. cit., in Philippe BOURDIN (dir.), op. cit., p. 231-232.

101. «En dis ans, la Révolution a parcouru tout l'éventail des formes politiques connues. Comment les Français n'auraient-ils pas été convaincus, la roue ayant fait un tour complet, de l'imminence d'une restauration?»: Patrice GuENIFfeY, Le Dix-huit Brumaire. L'épilogue de la Révolution française, Paris, Gallimard, 2008, p. 240.

102. Moniteur, XX, n. 124, [23/1/1799], République française paris le 3 pluviose VII, Directoire exécutif, p. 503-504.

103. Cfr. Luca scuccimarRA, "Dalla Costitutione dell'anno III a quella dell'anno VIII: rottura e continuità ", in Antonino De Francesco (a cura di), Da Brumaio ai Cento giorni. Cultura di governo e dissenso politico nell'Europa di Bonaparte, Milano, Guerini e Associati, 2007, p. 103-155.

104. Moniteur, n. 298, octidi 28 messidor an VII [16/07/1799], Discours présenté par le citoyen Sieyès, président du directoire exécutif, à la fête du Quatorze Juillet, le 26 messidor, p. 1213.

105. Moniteur, n. 298, octidi 28 messidor an VII [16/07/1799], p. 1213.

106. Moniteur, n. 302, duodi, 2 thermidor an 7 [20/07/1799], Suite de la séance du 26 messidor, p. 1227-1228.

107. Moniteur, XXI, n. 315, 15 thermidor an VII, Séance du 9 thermidor an VII, p. 1280-1281.

108. Moniteur, XXI, n. 328, 28 thermidor an VII, Conseil des anciens, séance du 26 thermidor VII, p. 1333.

109. Moniteur, XXI, n. 351, 21 fructidor an VII, République française, Paris, le 20 frimaire, Le directoire exécutif aux français, du 17 fructidor, an 7, p. 1424.

110. Moniteur, XXI, n. 314, 14 thermidor an VII, République française, p. 1276-1277. Per un'analisi di questi testi si vedano Pierre SERNA, art. cit., in Philippe BOURDIN, op. cit., p. 220 e ss. e Olivier LUTAUD , Des Révolutions d'Angleterre à la Révolution Française. Le tyrannicide et Killing no murder (Cromwell, Athalie, Bonaparte), Martinus Nijoff, La Haye, 1973, p. 245-246 (ora in Id., art. cit., p. 604-607). Per una visione completa sull'uso della storia inglese in Constant si rinvia invece a Geoffrey CUBITT, «Revolution, Reaction, Restoration: The Meanings and Uses of Seventeenth-Century English History in the Political Thinking of Benjamin Constant (1797-1830) », in European Review of History, vol. 14, 1, March 2007, p. 21-47.

111. Recensendo il pamphlet di Constant e comparandolo a quello di Boulay, la Décade ne invoca un altro che dovrebbe far emergere le differenze tra le due rivoluzioni: «Il reste un troisième ouvrage à faire sur le même sujet; c'est celui où l'on montrera la différence des principes sur lesquels se fondent les deux révolutions, et ce que les progrès des lumières dans toute l'Europe, les circonstances qui ont amené chacune de ces révolutions et qui les accompagnent, mettent de 
distance entre-elles »: La Décade, philosophique, littéraire et politique, n. 30, an VII ${ }^{\mathrm{e}}$ de la République Française, 30 messidor (18/07/1799), Variétés, p. 182-183.

112. Jean-Baptiste SALAVILLE, De la Révolution française comparée à celle de l'Angleterre, ou lettre au Répresentant du peuple Boulay (de la Meurthe), sur la différence de ces deux révolutions, Paris, prairial an VII, p. 41.

113. Ibidem, p. 30-40.

114. Constant riprovera a Boulay di aver mostrato « les crimes et les délire des oppresseurs de la république anglaise » e di aver passato sotto silenzio quelli dei " restauratori della regalità » ( Préface, p. III). Per rimediare, si propone di raccontare « ce qui suivit la chûte de la république en Angleterre »: « Je dirai comment Charles II, après avoir tout fait pour rassurer les Anglais, trompa misérablement ceux qui s'étoient lassiés rassurer par lu i » (p. VI).

115. Senza dimenticare, però, che lo stesso Constant si era già soffermato sulla diversità tra le due rivoluzioni nell'anno V, con la pubblicazione di due saggi: De la force du gouvernement actuel de la France et de la nécessité de s'y rallier (1796) e Des réactions politiques (1797). Su questo si veda Mario LEONARDI, art. cit., p. 157-158.

116. Madame DE STAËL, Des circonstances actuelles qui peuvent terminer la Révolution et des principes qui doivent fonder la République ne France, in Lucia Omacini (dir.), Euvres complètes, III, 1, Paris, Champion, 2009, p. 330-331. Sull'immagine dell'Inghilterra seicentesca in Madame de Staël, si vedano le considerazioni di Bronislaw BACzKo (Ibidem, Introduction. Opinions des vainqueurs, sentiments des vaincus, p. 189) e di Mario LEONARDI, art. cit., p. 156-158.

117. Moniteur, XXI, n. 314, 14 thermidor an VII, République française, p. 1276-1277.

118. Cfr. Pierre SERNA, art. cit., in Philippe BOURDIN (dir.), op. cit., p. 230.

119. Riprendo questa definizione da Pierre SERNA, art. cit., in Philippe BOURDIN (dir.), op. cit.: sulle affinità tra Boulay e Sieyès si veda Ibidem, p. 226 e 235.

120. BOULAY DE LA MEURTHE, op. cit., p. 2-3.

121. Per una ricostruzione esaustiva delle giornate di Brumaio, rinviamo a Luca Scuccimarra, op. cit., p. 11-50.

122. Bernard GAINOT, 1799, un nouveau Jacobinisme?, Paris, CTHS, 2001, p. 448.

123. Moniteur, XXI , n. 49, Conseil des anciens, séance du 18 brumaire an 8, p. 192.

124. «Le 18 Brumaire, déjà, au Conseil des Anciens, un sénateur, Dentzel, avait alerté ses pairs. Craignant la fin de la liberté, il voyait dans l'intervention de Bonaparte la marque d'un Cromwell ou d'un César »: Pierre SERNA, « "Du gouvernement du Lion ... ou règne de l'Astre brillant ?" Le 18 Brumaire au regard des historiens contemporains du premier Consulat (1800-1802) », dans JeanPierre Jessenne (dir.), Du Directoire au Consulat, t. 3, Brumaire dans l'histoire du lien politique et de l'État-Nation, Lille, Presses Universitaires du Septentrion, 2001, p. 353. Cfr. anche Patrice GUENIFFEY ,op. cit., p. 250-251.

125. Moniteur, XXI, n. 49, 19 brumaire an VIII, L'écrit suivant a été distribué, ce matin à neuf heures, autour des conseils. Dialogue entre un membre du conseil des anciens et un membre du conseil des cinqcents, p. 190. Baczko attribuisce tale scritto ad un personaggio interno alla cerchia bonapartista come Roederer (Bronislaw BACZKO, Politiques de la Révolution française, Gallimard, Paris, 2008, p. 536-541).

126. Patrice GUENIFFEY, op. cit., p. 22-28.

127. Moniteur, n. 51, Primidi, 21 brumaire an 8, Corps Législatif séant à Saint-Cloud, Conseil des Anciens, présidence de Lemercier, séance du 19 brumaire, p. 198.

128. Ibidem, p. 198-199. Si veda anche un altro resoconto del Moniteur, XXI, n. 50, 20 brumaire an VIII, Saint-Claud, le 19 brumaire, 8 heures du soir, p. 196.

129. Moniteur, n. 50, décadi, 20 brumaire an 8 , Corps Législatif séant à Saint-Cloud, Conseil des CinqCents, présidence de Lucien Bonaparte, séance du 19 brumaire, p. 194.

130. Ibidem, p. 195-196. 
131. Moniteur, XXI, n. 50, 20 brumaire an VIII, Saint-Claud, le 19 brumaire, 8 heures du soir, p. 196.

132. Sulla « leggenda dei pugnali », si veda ancora Scuccimarra, op. cit., p. 29-30. Per la versione di Bonaparte cfr. il suo resconto pubblicato dal Moniteur il 28 brumaio.

133. Moniteur, 53, tridi 28 brumaire an VIII, Proclamation du général en chef Bonaparte, le 19 brumaire, onze heures du soir, p. 205-206.

134. «Il y eut une violente et courte échauffourée [...] Aucun n'avait eu le réflexe de Tallien qui, le 9 thermidor, avait brandi à la Convention un poignard - dont il n'avait du reste pas l'intention de se servir - en jurant qu'il se tuerait plutôt que de se soumettre à Robespierre »: Patrice GUENIFFEY, op. cit., p. 292.

135. « On connait les étapes qui conduisirent Napoléon du consulat provisoire à l'empire. Il convient d'insister sur la prudence avec laquelle Napoléon a procédé pour établir le régime impérial »: Jean TULARD, « Napoléon: la continuité romaine », in Da Roma alla terza Roma. Documenti e studi, 1983, p. 225-226.

136. «L'Empereur lui-même ne cessera d'ailleurs de rejeter l'assimilation infamante. Ainsi, à Sainte-Hélène, il explique au général Bertrand : 'La Révolution d'Angleterre n'a rien de commun avec la nôtre, et Cromwell rien de commun avec moi ; il a tout fait par la force, et moi tout régulièrement par les lois »: Roger BARNY, art. cit., p. 397.

137. Oppure, quando avrà letto la riedizione (anno IX e anno X) del Précis historique sur Crumwell di L'Espinasse de Langeac, il Parallèle de la révolution d'Angleterre en 1642 et de celle de France (1800) di Nougaret e l'Histoire philosophique et politique des Révolutions d'Angleterre (anno VII,1799) di Jean Chas. Su tutto si veda Olivier LUTAUD, Des Révolutions d'Angleterre à la Révolution Française. Le tyrannicide et Killing no murder (Cromwell, Athalie, Bonaparte), Martinus Nijoff, La Haye, 1973, p. 245-246 (ora in Id., art. cit., p. 604-607).

138. Le code des tyrannicides, adressé à tous les peuples opprimés, Lyon, an VIII (1800).

139. Sul tema del tirannicidio e dell'opposizione a Napoleone all'inizio del Consolato, si veda anche Bernard GAINOT, «La République contre elle-même. Figures et postures de l'opposition à Bonaparte au débout du Consulat (novembre 1799-mars 1801) », in Antonino DE FRANCESCO (a cura di), Da Brumaio ai Cento giorni. Cultura di governo e dissenso politico nell'Europa di Bonaparte, Milano, Guerini e Associati, 2007, p. 143-155.

140. Pierre SERNA, “Du gouvernement du Lion ... ou règne de l'Astre brillant?", art. cit., p. 352, nota 21.

141. Mémoires de M. de Bourrienne, ministre d'état, sur Napoléon, le Directoire, le Consulat, l'Empire et la Restauration, tome IV, Paris, 1829 , p. 218. Si veda anche la testimonianza di Roederer riporata da Fierro (Les français vus par eux-mêmes. Le Consulat et l'Empire, Paris, Robert Laffont, 1998, p. 1087) e commentata da Thierry LENTZ, Le Grand Consulat, 1799-1804, Paris, Fayard, 1999, p. 266-267.

142. Una strategia, quest'ultima, che ovviamente Napoleone non portava avanti da solo. Jean Chas, ad esempio, che negli ultimi mesi dell'anno VII (1799) aveva pubblicato un'Histoire philosophique et politique des révolutions d'Angleterre, depuis la descente de Jules-César jusqu'à la paix de 1783, a marzo dell'anno successivo si è prodigherà per smentire i parallelismi pericolosi allora in circolazione tra Bonaparte, Cesare e Cromwell (Sur Bonaparte Premier consul de la République française, par le citoyen J. Chas, Paris, ventôse, an VIII [marzo 1800], p. 27 e 49-52).

143. Ho discusso altrove questa tesi formulata da ultimo da François HARTOG, op. cit.: Daniele Di BARTOLOMEO, « Lo specchio infranto. "Regimi di storicità" e uso della storia secondo François Hartog ", in Storica, vol. 49, 2011, p. 63-94.

144. Michael SONENSCHER, Sans-culottes. An Eighteenth-Century Emblem in the French Revolution, Princeton and Oxford, Princeton University Press, 2008, p. 367.

145. «La conception classique de l'histoire comme décadence et de la révolution comme retour ne disparut pas. Conception cyclique et conception cumulative de l'histoire coexistaient»: Patrice GUENIFFEY, La politique de la Terreur. Essai sur la violence révolutionnaire, Paris, Fayard, 2000, p. 44. 
146. Cfr. Jean-Marie GOULEMOT, Discours, révolution et histoire (Représentations de l'histoire et discours sur les révolutions de l'Age Classique aux Lumières), Paris, UGE, 1975; Id., Le règne de l'histoire. Discours historiques et révolutions (XVII'-XVIII ${ }^{e}$ siècle), Paris, Albin Michel, 1996, p. 389-395.

\section{RIASSUNTI}

Cet article s'intérese à l'usage de l'histoire de l'Angleterre entre 1649 et 1660 durant la période du Directoire. L'analyse est centrée sur le précédent anglais. La démonstration s'appuie sur la reconstruction du récent débat historiographique sur républicanisme et Révolution française, en tenant compte des textes anglais lus, traduits et discutés par les hommes du Directoire. Comment à 150 ans d'écart vont jouer les différences et les rapprochements entre les deux pays, et qu'en tirer pour l'analyse d'une révolution interminable ou d'une république difficile à stabiliser? Comme dans l'Angleterre de 1660, la menace d'un général plane.

This article is dedicated to the use of the English republican history (1649-1660) in France, from the fall of Robespierre to the first anniversary of the Napoleon's rise to power (1794-1800). The analysis focuses on the role played by the English precedent during the crucial junctures that have marked the existence of the French Republic, from Thermidor to end of the Directory. This part is preceded by a critical review of the recent historiographical debate on the link between Republicanism and French Revolution and it concludes with a brief reflection on the consequences induced by the revolutionary experience on the ideas of revolution and republic.

\section{INDICE}

Mots-clés : usage politique de l'histoire, Révolution anglaise, républicanisme, Thermidor, Directoire, Napoléon Bonaparte

\section{AUTORE}

\section{DANIELE DI BARTOLOMEO}

Assegnista di ricerca

Facoltà di Scienze della Comunicazione

Università degli Studi di Teramo

ddibartolomeo@unite.it 